\title{
Effects of Local Soil Conditions on the Topographic Aggravation of Seismic Motion: Parametric Investigation and Recorded Field Evidence from the 1999 Athens Earthquake
}

\author{
by Dominic Assimaki, George Gazetas, and Eduardo Kausel
}

\begin{abstract}
During the 1999 Athens earthquake, the town of Adàmes, located on the eastern side of the Kifissos river canyon, experienced unexpectedly heavy damage. Despite the particular geometry of the slope that caused significant motion amplification, topography effects alone cannot explain the uneven damage distribution within a 300-m zone parallel to the canyon's crest, which is characterized by a rather uniform structural quality. In this article, we illustrate the important role of soil stratigraphy and material heterogeneity on the topographic aggravation of surface ground motion. For this purpose, we first conduct an extensive time-domain parametric study using idealized stratified profiles and Gaussian stochastic fields to characterize the spatial distribution of soil properties, and using Ricker wavelets to describe the seismic input motion; the results show that both topography and local soil conditions significantly affect the spatial variability of seismic motion. We next perform elastic two-dimensional wave propagation analyses based on available local geotechnical and seismological data and validate our results by comparison with aftershock recordings.
\end{abstract}

\section{Introduction}

It has been long recognized that topography can significantly affect the amplitude and frequency characteristics of ground motion during seismic events. Documented observations from destructive seismic events have shown that buildings located at the tops of hills, ridges and canyons suffer more intensive damage than those located at the base: the Lambesc earthquake (France 1909), the San Fernando earthquake (1971), the Friuli earthquake (Italy 1976), the Irpinia earthquake (Italy 1980), the Chile earthquake (1985), the Whittier Narrows earthquake (1987), the "Eje-Cafetero" earthquake (Colombia 1998), and recent earthquakes in Greece (Kozani 1995 and Athens 1999) and Turkey (Bingöl 2003) are only some examples of catastrophic events, during which severe structural damage was reported on hilltops or close to steep slopes. Among several recorded motions that provide evidence on topographic amplification, two classical examples are of particular note: (1) the peak ground acceleration (PGA) $1.25 \mathrm{~g}$ recorded on a sharp ridge near the $\mathrm{Pa}-$ coima Dam abutment during the San Fernando earthquake of magnitude 6.4 defined in the Richter scale $\left(M_{\mathrm{L}}\right)$, and (2) the PGA $=1.8 \mathrm{~g}$ record obtained on a flat, broad hill in Tarzana during the $M_{\mathrm{L}} 6.8$ Northridge earthquake.

Instrumental studies performed in recent years provide confirmation of the macroseismic observations. They indicate systematic amplification of seismic motion over convex topographies such as hills and ridges, deamplification over concave topographic features such as canyons and hill toes, and complex amplification and deamplification patterns on hill slopes that result in significant differential motions. These studies quantify directly crest-to-base amplification ratios by using dense instrumented arrays, yet they are generally limited to low-amplitude recordings of aftershock sequences or microtremors (Celebi, 1987; Pederson et al., 1994).

Prompted by observational and instrumented evidence, the problem of scattering and diffraction of seismic waves by topographical irregularities has been studied often, but with a focus mostly on two-dimensional simulations of isolated ridges or depressions on the surface of homogeneous, linearly elastic half-spaces. Boore (1972) modeled the effects of a ridge by using finite differences, Smith (1975) utilized finite-element methods, Sanchez-Sésma and Campillo (1991) used boundary-element methods, and Bouchon (1973) and Bard (1982) used a discrete-wavenumber method to simulate topographic wave diffraction. In a thorough review of published results, Geli et al. (1988) noted that, in almost all these models, crest-to-base amplification factors of peak acceleration are on the order of two for an isolated ridge.

A limited number of examples, which involve more complex numerical simulations, can be found in Bard and Tucker (1985), who investigated the antiplane response of a 
ridge chain with irregular subsurface layering, and SánchezSesma (1983) and Bouchon et al. (1995), who investigated the response of three-dimensional homogeneous ridges. Also, Geli et al. (1988) evaluated the effects of compositional layering and complex topography. They noted a complex pattern of amplitude fluctuations that varied with the location on the ridge and the degree of sediment cover. To expand on this idea, Deng (1991) developed a numerical model, in which the geometry and geology of the configuration are simulated. Ashford et al. (1997) used this numerical model to illustrate the significance of steep slopes in site amplification.

Geli et al. (1988) and Bard (1999) compiled instrumental and theoretical results and reported that there is qualitative agreement between theory and observations on topography effects. Nonetheless, from a quantitative viewpoint, their study suggests that a clear discrepancy still exists in numerous cases, where the observed amplifications are significantly larger than the theoretical predictions. As an example, numerous observations of spectral amplifications are larger than 10 , but only very few predictions by numerical models are of such amplitude.

In this article, we show that the soil stratigraphy and heterogeneity do also affect the topographic aggravation of ground motion near the vertex of clifftype topographies; it is therefore necessary to model simultaneously the geometry of the topographic irregularity and the local soil conditions to obtain accurate estimates of the amplification level. For this purpose, we first review published results on the response of topographic irregularities, underlain for the most part by homogeneous half-spaces. By means of elastic parametric simulations, we then illustrate the effects of soil stratigraphy and material heterogeneity on the ground-motion amplification for cliff-type topographies. We assess the role of soil conditions by computing the motion amplification in the vicinity of the asperity that occurs in excess of the farfield surface response; note that the latter corresponds to the seismic input already filtered by the near-surface geological formations.

Based on detailed topographic surveys, local geotechnical investigations, and available acceleration recordings from the Athens 1999 event, we conduct elastic simulations for the local-site conditions. Finally, we validate our conclusions by comparison with aftershock recordings of the event. It should be added that this article focuses on elastic ground-shaking effects, which are primary related to wave propagation phenomena. The importance of site effects associated with soil nonlinearity and large inelastic deformations are not considered herein, but will be taken up in a forthcoming article by the authors.

\section{Surface Topography Effects: Theory and Observations}

Acceleration signals recorded in the past three decades reveal that subsoil characteristics can have significant influ- ence on the amplitude level, the frequency composition, and the duration of shaking. Although the effects of the surface geology are usually described as soil amplification, remember that soil filtering can also depress harmonic components of the incident seismic waves, in which case deamplification of shaking takes place.

In general, seismic topography effects may be elicited by discontinuities in elevation, convex and concave topographic irregularities (hills, ridges, canyons, cliffs, and slopes), complicated subsurface conditions (sedimentary basins, alluvial valleys), and lateral discontinuities in geology (faulting, debris zones). Of these, the effects of hills and canyons are the most pervasive, so they are also the most frequently studied by means of numerical and theoretical models, which predict (1) systematic amplification of seismic motion on ridge crests and convex topographies such as cliffs; (2) deamplification over concave topographic features such as canyons and hill toes; and (3) complex amplification and deamplification patterns on hill slopes that result in significant differential motions. These effects are shown to be rather sensitive to the characteristics of the incident wave field, namely the wave type, the incidence angle, and the azimuth of propagation. According to Bard (1999), they are related to three physical phenomena:

1. The sensitivity of the surface motion to the incidence angle, which is especially large for $S V$ waves near the critical angle. The slope angle thus produces significant variations in surface motions (Fig. 1). Kawase and Aki (1990) suggested that this effect was a contributing cause to the peculiar damage distribution observed on a mild slope during the Whittier Narrows, California, earthquake of 1987.

2. The focusing or defocusing of seismic waves reflected along the topographic surface. Sanchez-Sesma (1990)

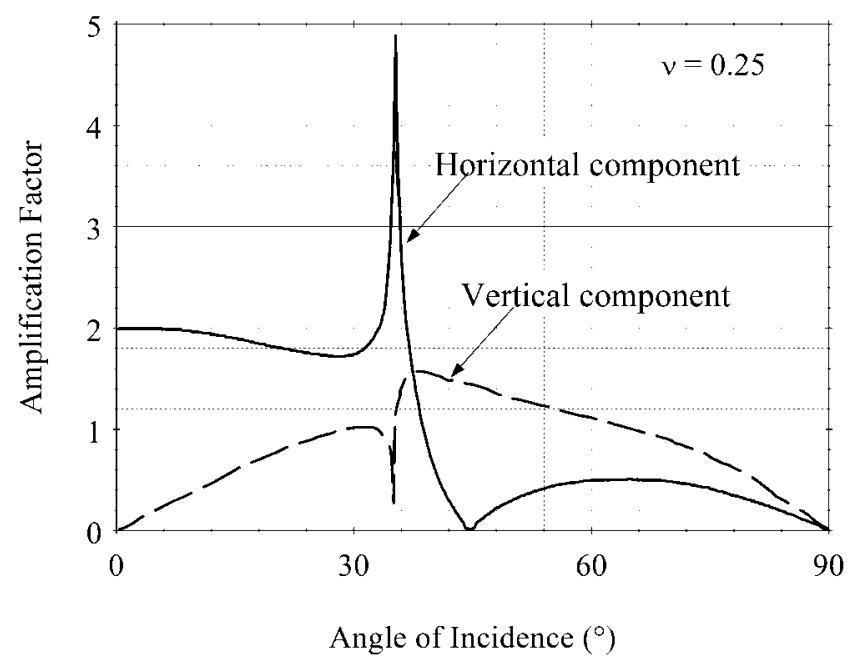

Figure 1. Sensitivity of surface motion to incidence angle for obliquely incident plane $S V$ waves (Poisson's ratio, $v=0.25$ ). 
provided insights into this effect by means of a wedgeshaped medium. Nonetheless, instrumental proof of such focusing/defocusing effects in strong seismic shaking has not been adequate because of the scarcity (or absence) of strong-motion arrays in the neighborhood of distinct topographic features.

3. The diffraction of body and surface waves that propagate downward and outward from the topographic feature and lead to interference patterns between the direct and diffracted waves. However, these diffracted waves generally have smaller amplitudes on the surface than the direct body waves, at least for smooth natural topographies. Such interference patterns consistently predicted by theory have been reported by Pedersen et al. (1994) from semidence array recording in Greece, where the amplitude of the outwardly going waves was shown to be about one-fifth of that of the primary wave.

Studies also show that the estimated amplification is lower for incident $P$ waves than for incident $S$ waves and that it is slightly larger for in-plane horizontal motion ( $S V$ waves) than for antiplane motion ( $S H$ waves). Nonetheless, time-domain crest-to-base amplification ratios from theoretical or numerical studies do not exceed-with a few exceptions - the value of 2 , underestimating considerably the amplification values as high as 10 observed during microtremors in the field.

\section{Theoretical and Numerical Studies}

Incident Wave-Type. The incidence of $\mathrm{SH}$ waves has been studied more frequently because of its mathematical simplicity, inasmuch as reflection and diffraction of $S H$ waves in two dimensions does not involve conversion into other wave types. By contrast, $S$ and $P$ waves involve mode conversions into one another that often result in amplification values exceeding those for $S H$ waves. More specifically, all studies show that a zone exists in the vicinity of a slope where high amplification of the incident seismic motion occurs because of the combination of primary $S V$ and diffracted Rayleigh waves (Bouchon, 1973; Boore et al., 1981; Ohtsuki and Harumi, 1983; Ashford and Sitar, 1997).

Direction of Incident Waves. The direction of the incident seismic waves is described by the vertical angle of incidence $(\theta)$ and the azimuth of propagation. Many authors such as Trifunac (1973), Wong and Trifunac (1974), Wong and Jennings (1975), Sills (1978), Sanchez-Sésma and Rosenblueth (1979), England et al. (1980), and Bard (1982), have investigated the displacement field over topographic features acted upon by oblique and grazing $S H$ plane waves. Investigations have been performed for both $P$ and $S V$ waves, which have demonstrated the great sensitivity of the $P-S V$ coupling to the incidence angle, especially in the $S V$ waves with near critical incidence (Fig. 1).

The most striking feature of the diffracted field is the directivity effect resulting from forward-scattered Rayleigh waves that show the same qualitative behavior for $S H, S V$, and $P$ incidence (Bard, 1982). The strengthening of the forward-scattered Rayleigh wave in the $P$ case has also been experimentally verified by Rogers et al. (1974). Published site-specific analyses show, however, that even if topographic amplification phenomena are more pronounced for obliquely incident waves, the absolute magnitude of acceleration at the crest (both horizontal and vertical) is, in general, larger for vertically propagating waves.

Frequency Content of Incident Motion. Among others, Ohtsuki and Harumi (1983), Buchbinder and Haddon (1990), Aki (1988), and Ashford et al. (1997) have shown that topography effects are more pronounced for wavelengths comparable with the geometric characteristics of the irregularity. These studies also showed that topographic amplification is negligible for wavelengths significantly longer than the dimensions of the asperity.

Topographic Shape. The problem of seismic amplification as a function of the topographic shape of surface irregularities has been studied extensively. We shall refer to a few characteristic examples that can be readily compared with the numerical simulations in the ensuing: (1) Boore et al. (1981), who studied the effects of a cliff slope on the dispersion of the incident waves; (2) Ashford and Sitar (1997) studied the response at the crest of cliffs as a function of the slope inclination $\left(30^{\circ}, 45^{\circ}\right.$, and $\left.90^{\circ}\right)$ for vertically propagating $S V$ and $S H$ waves; (3) Bouchon (1973) studied the effects of the topographic shape on the surface response of ridges and canyons subjected to incident SH waves; (4) Hudson (1967) and Hudson and Boore (1980) related the amplitude of the diffracted wave with the spatial Fourier transform of the topographic cross section, based on published numerical studies and the theoretical calculations of Gilbert and Knopoff (1960). Finally, published results on the response of shallow canyons or low ridges (where the interaction of scattered wave fields due to the simultaneous presence of two cliffs could be neglected) could also be used to assess qualitatively the spatial distribution of amplitude along the surface behind the crest of a ridge.

\section{Experimental Results}

Davis and West (1973) recorded several aftershocks of the San Fernando 1971 earthquake at the base and crest of Kagel Mountain and Josephine Peak, California. They also compared seismic signals that were caused by an underground cavity collapse and were recorded at the base and crest of Butler Mountain, Nevada. In each case, they computed the crest-to-base ratios of pseudovelocity response spectra (5\% damping) and observed a large, frequencydependent amplification. In general, the wavelengths that corresponded to these amplification frequencies were found to be comparable with the mountain width, which is in good 
agreement with theoretical results. Nonetheless, observed amplification levels both in the time and frequency domain were much larger than the values predicted by theoretical models; even greater discrepancies were reported for spectral amplification values.

Rogers et al. (1974) recorded an underground nuclear explosion at several sites across the NASA Mountain in Nevada. They performed only time-domain comparisons of crest and base motions, and the observed amplifications are about $25 \%$, which is in good agreement with theoretical predictions. As in the theoretical and laboratory models, the NASA Mountain has a rather smooth and regular twodimensional geometry, and the incident wavefront was parallel to the ridge axis.

Griffiths and Bollinger (1979) conducted a field program in the Appalachian Mountains, where they recorded 137 events at the base and crest of six topographic features. Only one event was natural; the other events were quarry and mine blasts. The instruments were smoked-paper recorders, and crest-to-base comparisons could be made only in the time domain. Once again, the seismic motion was observed to be systematically larger at the top than at the base. For a given crest-to-base pair of stations, amplification ratios obtained for different events exhibited larger scatter than intraevent recordings from different pairs, but the average values ranged between $170 \%$ and $340 \%$, which is significantly larger than the theoretical predictions. In this case, inconsistency between experimental data and theory was attributed to the presence of a surface soft layer not simulated in the analyses and to the interaction between subparallel ridges with quasi-periodic patterns as opposed to the simulated isolated ridge.

Tucker et al. (1984) measured the spectral differences between seismic records that were obtained at two-dimensional hard-rock sites. For a small ridge, they observed that the spectral ratios were similar, irrespective of the azimuth, distance, and size of the seismic events. They also reported that the spectral amplification computed with respect to a nearby tunnel, not with respect to the ridge base, reached values as high as 8. A discussion of the inconsistency between observations and simple theoretical models can be found in Bard and Tucker (1985).

Finally, Umeda et al. (1996) recorded aftershocks of the Western Nagana Prefecture Earthquake, 1984, on a small (100 m high, $500 \mathrm{~m}$ wide) topographic feature, where thrown-off boulders gave evidence of unusually high accelerations during the main shock. They observed crest-to-base spectral amplifications larger than 10, which were again significantly larger than the theoretical predictions. Discrepancies in this case were attributed to the nonhomogeneous local geological structure, despite the nearly twodimensional geometry of the ridge is.

Based on the compilation of published instrumental and theoretical studies, the conclusions drawn by Geli et al. (1988) and revisited by Bard (1999) are summarized here. First, a qualitative agreement exists between theory and ob- servations about the existence of seismic-motion amplification at ridges and mountain tops and deamplification at the base of hills. The amplification is generally larger for horizontal components (roughly corresponding to $S$ motion) than for the vertical component (mostly $P$ motion). This amplification (or deamplification) phenomenon is frequency dependent. There is a satisfactory qualitative agreement between instrumental observations and theoretical results for the relation between geometrical and mechanical characteristics of a given topography and the frequency range where amplification is significant: the maximum effects correspond to wavelengths comparable with the horizontal dimension of the topographic feature. Second, from a quantitative viewpoint, however, clear discrepancies exist between theory and observations. Cases have been reported in which field measurements exhibit only very weak amplifications at ridge crests and fit very well the numerical results (Rogers et al., 1974). However, numerous cases also exist in which the observed amplifications are significantly larger than the theoretical predictions obtained from sophisticated, two- or three-dimensional models (Bouchon et al., 1995). Numerous observations have been made of spectral amplifications larger than 10 , but only very few predictions of such amplitudes have been made by numerical models. This has been confirmed by some recent instrumental studies in Greece, California, and the French Alps (Pedersen 1994; Nechtschein et al., 1995; Bouchon and Barker, 1996; Lebrun et al., 1999).

The focusing of seismic energy in convex topographies, as predicted by theoretical models, certainly plays a significant role in observed amplification effects. Nonetheless, the quantitative disagreement between theory and observations shows that it is not the only physical phenomenon involved. A case study from the Athens 7 September 1999 earthquake is indeed an example in which the simultaneous effects of topography and local soil conditions resulted in substantial amplification at the crest of a canyon. Documented macroseismic observations, recorded acceleration time histories from the seismic event, in conjunction with topographic survey and geotechnical investigation data, provide the necessary components for a detailed analysis on site effects.

\section{The Athens 7 September 1999 Earthquake}

The Athens, Greece, earthquake of 7 September, 1999 occurred at 11:56:50.5 UTC at the western bounds of the greater metropolitan area of Athens. This seismic event was the first ever reported to have caused casualties within the urban area of Athens and can certainly be regarded as the worst natural disaster in the modern history of Greece.

Greece is undergoing active extension in a back-arc setting because of the subduction of the African plate beneath the Eurasian plate at the Hellenic Arc. In regions such as the Gulf of Corinth, just a short distance from the epicenter of the Athens earthquake, damaging, moderate-magnitude shocks are relatively frequent. Nonetheless, few damaging 
earthquakes have been documented in the epicentral region, with the 1981 Gulf of Corinth earthquakes being the most recent to cause damage in Athens (Psycharis et al., 1999).

During the mainshock, no fault showed clear evidence of rupture propagation to the free surface in the epicentral region. As a result, great uncertainty and confusion arose regarding the triggering mechanism of the event. The fault that caused the major seismic event of September 1999 was identified as being the Fili fault by means of a multidisciplinary approach that used the following techniques: (1) field observations, mapping, and fault measurements along the three main faults of the epicentral area; (2) morphotectonic approaches supplemented by 20-m digital elevation models; (3) landsat imagery interpretation; (4) macroseismic effects evaluation; and (5) aftershock-distribution data and focalmechanisms solution of the mainshock. Additional data came from radon soil emission measurements some days after the earthquake, which are concentrated mainly along the eastern edge of the Fili fault. Detailed seismological information can be found in Stavrakakis (1999), Delibasis et al. (2000), Kontoes et al. (2000), Rondogianni et al. (2000), Tselentis and Zahradnik (2000a, 2000b), and Pavlides et al. (2002). The projection of the rupture on the ground surface, along with the geography of the heavily damaged region, are schematically illustrated in Figure 2.

The modeling of teleseismic $P$ and $S H$ waves provided a well-constrained focal mechanism of the mainshock with strike of $105^{\circ}$ and dip of $55^{\circ}$. The rupture nucleated at $38.105^{\circ} \mathrm{N}, 23.565^{\circ} \mathrm{E}$, approximately $20 \mathrm{~km}$ northwest of the city of Athens, at a focal depth of $8 \mathrm{~km}$; subsequently, the rupture propagated upward, with the number of aftershocks being greater in the eastern part of the fault, that is, the part closer to Athens (Papadimitriou et al., 2002). One would therefore expect forward-directivity effects to have been present in the ground motions experienced at sites located to the east of the rupture zone, at distances within a few kilometers; such is the site studied in this article. (Note that whereas forward-directivity effects are well documented mostly with strike-slip and thrust-fault events, evidence of their importance in normal-fault moderate-magnitude events is also available. See Gazetas et al. (1990) and Gazetas (1996) for the Kalamata $M_{\mathrm{s}} 6$ earthquake of 1986 and the Aegion $M_{\mathrm{s}} 6.2$ earthquake of 1995, in Greece.) Finally, the rupturing process terminated beneath the Aegaleo Mountain range, whose orientation is nearly perpendicular to the strike of the activated fault. The mountain seems to have acted as a barrier, and the sudden interruption and possible turning of the rupturing process would have an effect similar to an impact, generating high-acceleration high-frequency waves.

Despite its moderate magnitude of $M_{\mathrm{s}} 5.9$ and the medium focal depth of $8 \mathrm{~km}$ (Papadopoulos et al., 2000), the seismic event resulted in the loss of 143 lives, the collapse of about 200 residential and industrial buildings, and the severe damage of another 13,000. The geographical distribution of buildings, the collapse of which caused fatalities, is indicative of the intensity of damage in the various towns

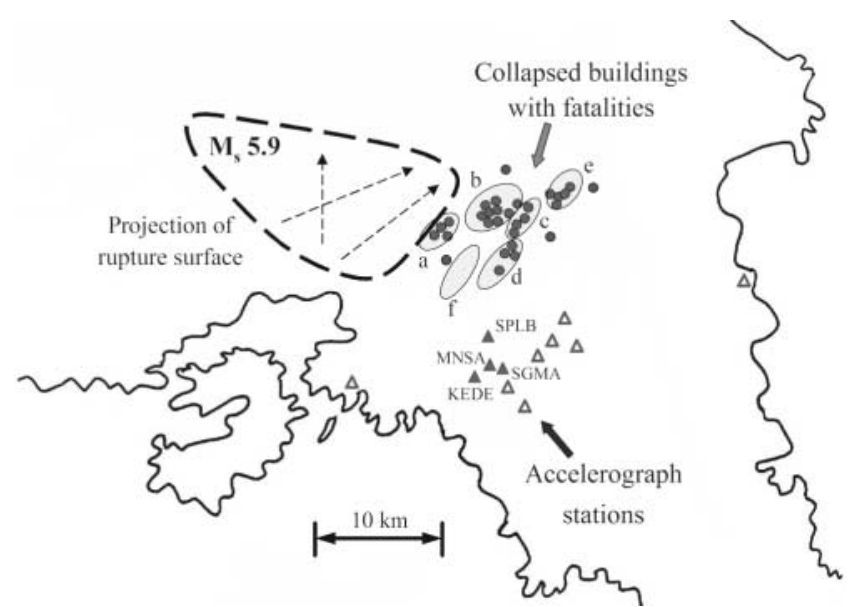

Figure 2. Sketch of the map of the earthquakestricken region showing the surface projection of the fault. The triangles indicate the location of accelerograph stations, four of which (shown with filled triangles) are utilized in our study. The black dots show the location of the 28 collapsed buildings with human casualties, indication of the damage intensity in the meizoseismal area; in areas a-e in particular, the maximum intensity in MMI was IX.

and regions; their location is depicted in Figure 2. In the town of Adàmes (area e in Fig. 2) studied in this article, at distance $8-9 \mathrm{~km}$ from the surface projection of the fault, the observed motion intensity varied in the Modified Mercalli Intensity scale $(\mathrm{MMI})=\mathrm{VII}^{+}-\mathrm{IX}$. In the same figure, we also indicate the locations of the four accelerograph stations that recorded the strongest motions during the event, namely KEDE, MNSA, SGMA, and SPLB.

Note that although the damage intensity reached MMI $=\mathrm{IX}$ in areas $\mathrm{a}, \mathrm{b}, \mathrm{c}$, and e, surrounding areas such as $\mathrm{f}$ sometimes suffered much less, with MMI not exceeding VII at mostly rocky sites (a difference of two units in MMI). The resulting damage distribution was described as strongly nonuniform in most of the hardest hit regions and towns. As a striking example, in the town of Menidi located at a 2 to 5$\mathrm{km}$ distance from the fault (denoted as b in Fig. 2), the damage varied from a catastrophic MMI = IX in the northern part of town to a modest $\mathrm{MMI}=\mathrm{VII}^{+}$south of the town center, a distance of less than $1 \mathrm{~km}$. Although numerous factors may have contributed to such nonuniformity, it is believed that local geotechnical and geological conditions exerted a major influence on the ground-motion characteristics and the resulting damage patterns.

From a structural viewpoint, the earthquake primarily affected low- and mid-rise buildings (two to five stories) in the broader area of Athens. Most of the damage occurred within $12 \mathrm{~km}$ of the epicenter and decreased rapidly with distance. In summary:

1. Severe damage was observed in areas 1 to $3 \mathrm{~km}$ from the epicenter, such as Ano Liosia (Fig. 2, area a) and Menidi (Fig. 2, area b). Obviously, the acceleration levels in these 
areas must have been high, considering the small distance from the epicenter. Unfortunately, no acceleration time histories were recorded in the meizoseismal area during the event.

2. Severe damage was also observed in scattered areas 5.0 to $10.0 \mathrm{~km}$ from the epicenter, such as Metamorfosi (Fig. 2, area c), Chelidonou (Fig. 2, area d), and Adàmes (Fig. 2, area e). In these areas, damages cannot be associated merely with the small epicentral distance. On the contrary, damage in these areas indicates the significance of additional factors causing amplification of the earthquake ground motion. In particular, soil conditions, topography effects, direction of rupture (directivity effects), and particularities of a significant underground geological structure of the bedrock in the broader area may have well contributed to the resulting damage pattern in the aforementioned areas.

3. The indications for local amplification of earthquake ground motion in recent deposits, resulting from the comparative examination of the geological and damagedistribution maps are strong, both in the epicentral area and further out.

4. In several cases, there is strong indication of the influence of topography on local amplification of ground motion. In particular, heavy damage occurred in buildings (especially industrial) located on the east bank of Kifissos River close to the Municipality of Adàmes (Fig. 2, area e).

The Town of Adàmes: Observations and Surface Topography

Adàmes (denoted as location e in Fig. 2) is a community of medium to low population density, roughly $1200 \mathrm{~m}$ in length and $300 \mathrm{~m}$ in width, lying next to the deepest canyon of the Kifissos river, the main river of the Athens metropolitan area. Overall, structural quality in Adàmes is judged as being not worse (or even perhaps better) than that of the buildings in towns located at equal or lesser distances from the source. Nonetheless, the collapse of three industrial and two residential buildings caused 18 deaths, in addition to a large number of injured. Many other buildings either collapsed or had their (usually soft, columns only) ground floor destroyed and were subsequently demolished. Their locations are depicted in Figure 3.

The location of the town next to the crest of the canyon, in conjunction with the high damage level, suggested that the local seismic intensity could be attributed to topography effects. Behind the crest, however, damage was bilaterally nonuniform, and along the axis parallel to the river, it was concentrated in two zones: one zone immediately next to the crest and another at a distance of about 200-300 m from it, as shown in Figure 3. Some scattered and less severe damage was observed at intermediate locations. It seems therefore, that factors other than the focusing of seismic energy at the vertex, must have contributed to the uneven distribution of damage along the crest of the canyon, which for practical purposes can be regarded as a two-dimensional topographic feature.

A topographic survey of the canyon, performed by the Survey Division of the National Technical University of Athens, March to April 2000, produced the cross-section $\mathrm{MM}^{\prime}$ shown in Figure 4a. Based on our preliminary simulations for the original canyon-type topography, which showed practically no interaction between adjacent slopes for vertically propagating incident waves and the dimensions of the riverbed, the slightly idealized geometry of a single cliff was used in our investigation, also shown in the same figure. The 40-m-deep and the nearly 2:1 (width/height) slope of the canyon cliff are also depicted in Figure 4b.

\section{Elastic Parametric Investigation}

We shall first identify which aspects of the canyon geometry may have aggravated the incident motion in the vicinity of the crest. We then investigate the role of soil layering and material heterogeneity by means of elastic parametric simulations. For this purpose, we perform twodimensional elastic wave propagation simulations in homogeneous, layered, and random media, using the finiteelement computer code DYNAFLOW (Prèvost, 1989, 1993) with appropriately fine discretization of the truncated computational domain and transmitting boundaries.

The numerical model consists of plane four-node quadrilateral and three-node triangular finite elements; their size is directed by the frequency content of the incident motion and the shear-wave velocity of the medium, to ensure detailed representation of the propagating wavelengths. Absorbing elements are placed around the discretized domain to avoid spurious reflections from the boundaries, and the input motion is prescribed in the form of effective forcing functions at the same nodes (to allow scattered waves to pass through the boundaries).

The model is subjected to seismic motion consisting of vertically incident $S V$ waves, which are prescribed at rock outcropping. The idealized input seismograms are initially taken in the form of Ricker waveforms, for simplicity. A schematic representation of the numerical model used in our investigation is shown in Figure 5. In all our simulations, the crest is defined at distance $x=300 \mathrm{~m}$ from the leftlateral boundary, beyond which the effects of the irregular surface topography were found to be negligible.

\section{Geometry of the Kifissos Canyon}

Figure 6 illustrates the wave field in the neighborhood of the Kifissos canyon when the latter is underlain by a homogeneous half-space and is acted on by vertically propagating $S V$ waves consisting of Ricker wavelets with normalized frequency $a_{0}=2 f_{0} H / V_{s}=1.00$, where $f_{0}$ is the central frequency of the pulse, $H$ is the height of the cliff, and $V_{s}$ is the shear-wave velocity of the half-space. The 


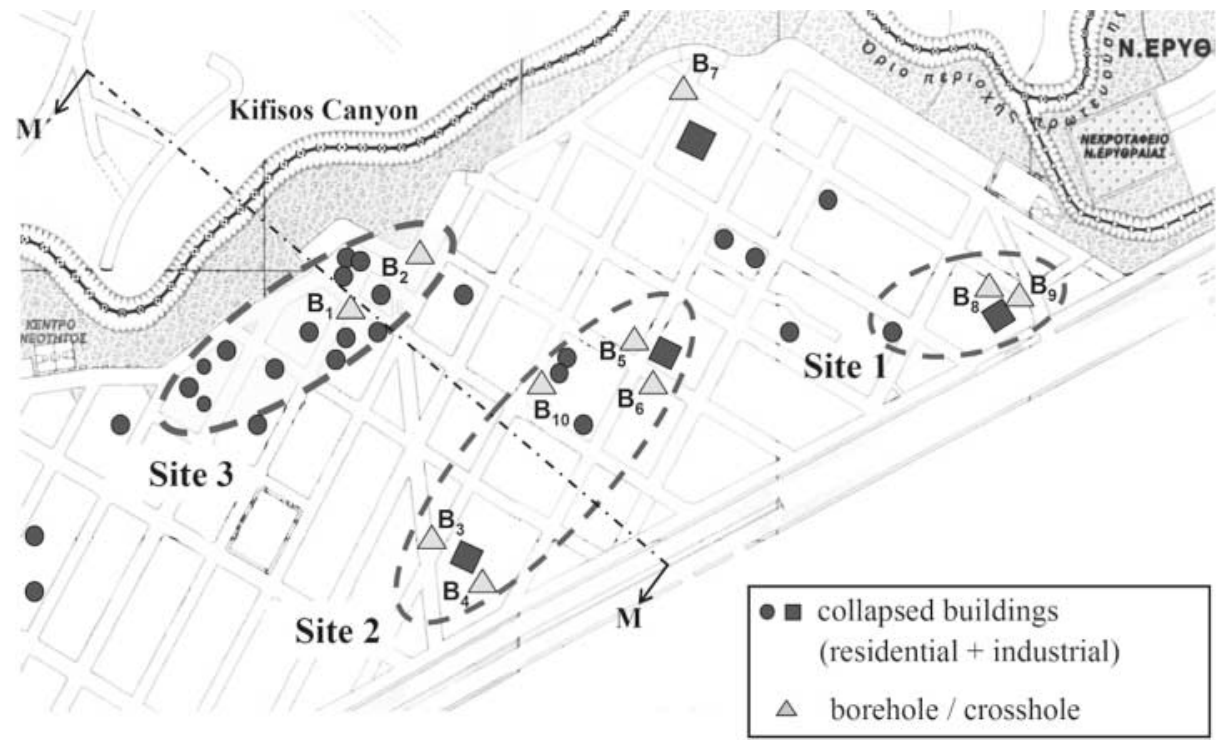

Figure 3. Plan view of Adàmes, showing the heavily damaged and collapsed residential (circles) and industrial (squares) buildings. Also shown are the locations of the geotechnical boreholes $\left(\mathrm{B}_{1}\right.$ to $\left.\mathrm{B}_{10}\right)$ and the topographic cross section $\mathrm{MM}^{\prime}$.

(a)

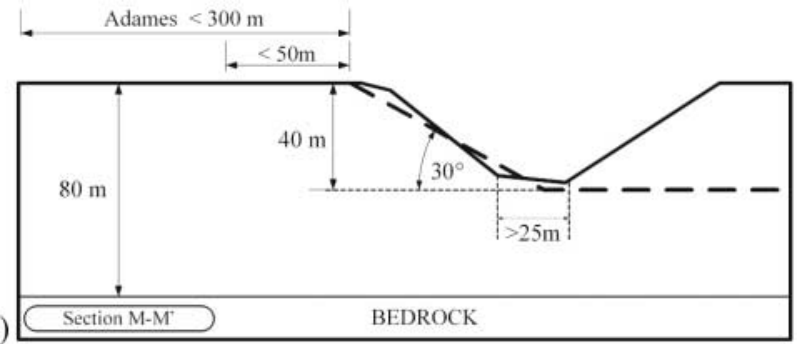

(b)

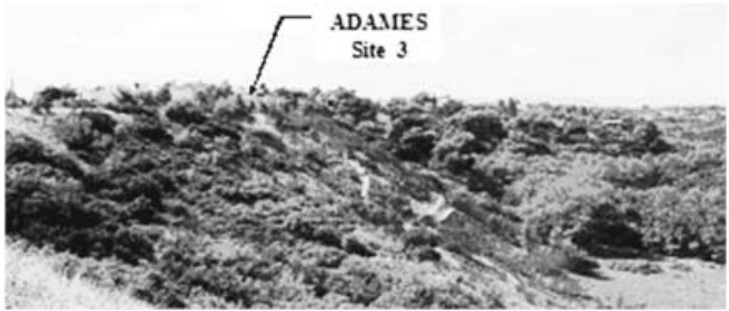

Figure 4. (a) Typical cross section of the topographic relief of the Kifisos river canyon and the region of Adàmes. Site 3 is located 10 to $50 \mathrm{~m}$ from the edge of the cliff, whereas site 2 is at about $300 \mathrm{~m}$. The idealized geometry used in our two-dimensional simulations is also shown (dashed line). (b) Bird's eye view of the cliff of the Kifissos canyon.

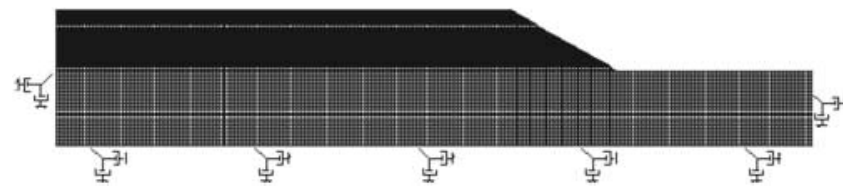

Figure 5. Typical two-dimensional finite-element model developed for the elastic parametric study of the problem.
Poisson's ratio of the elastic medium is $v=0.35$, typical of stiff clayey soils.

The direct/diffracted wave field shown in Figure 6 comprises the following waveforms: (1) direct $S V$ waves (denoted SV); (2) forward-scattered Rayleigh waves (denoted R1) generated at the boundaries of the shadow/illuminated zone at the lower corner of the cliff, propagating along the cliff and being forced to change direction at the upper corner; (3) backward-scattered Rayleigh waves (denoted R2) generated at the boundaries of the shadow/illuminated zone at the lower corner of the cliff and propagating outward; and (4) surface waves (denoted $S P$ ) that are generated along the cliff and propagate upward approximately with the $P$-wave velocity. As a result, these waveforms arrive in the vicinity of the crest almost simultaneously with the direct $S V$ wave.

The significant enhancement of forward-scattered Rayleigh waves (resembling forward-directivity effects), along with the presence of surface waves traveling along the slope with velocity of propagation that approaches the $P$-wave velocity can be explained from the combination of the slope angle and material Poisson's ratio corresponding to the present analysis. For $v=0.35$, critical incidence is calculated as:

$$
\theta_{\text {cr }}=\arcsin \left(V_{s} / V_{p}\right)=28.71^{\circ} \approx i=30^{\circ}
$$

Therefore, vertically propagating waves strike the free surface of the slope with almost critical incidence, resulting in the transformation of practically all the incident energy into surface waves that travel along the slope and constructively interfere with the direct $S V$ waves that arrive behind the crest. Therefore, the site conditions in Adàmes satisfy $a$ priori conditions that favor a complicated and detrimental 

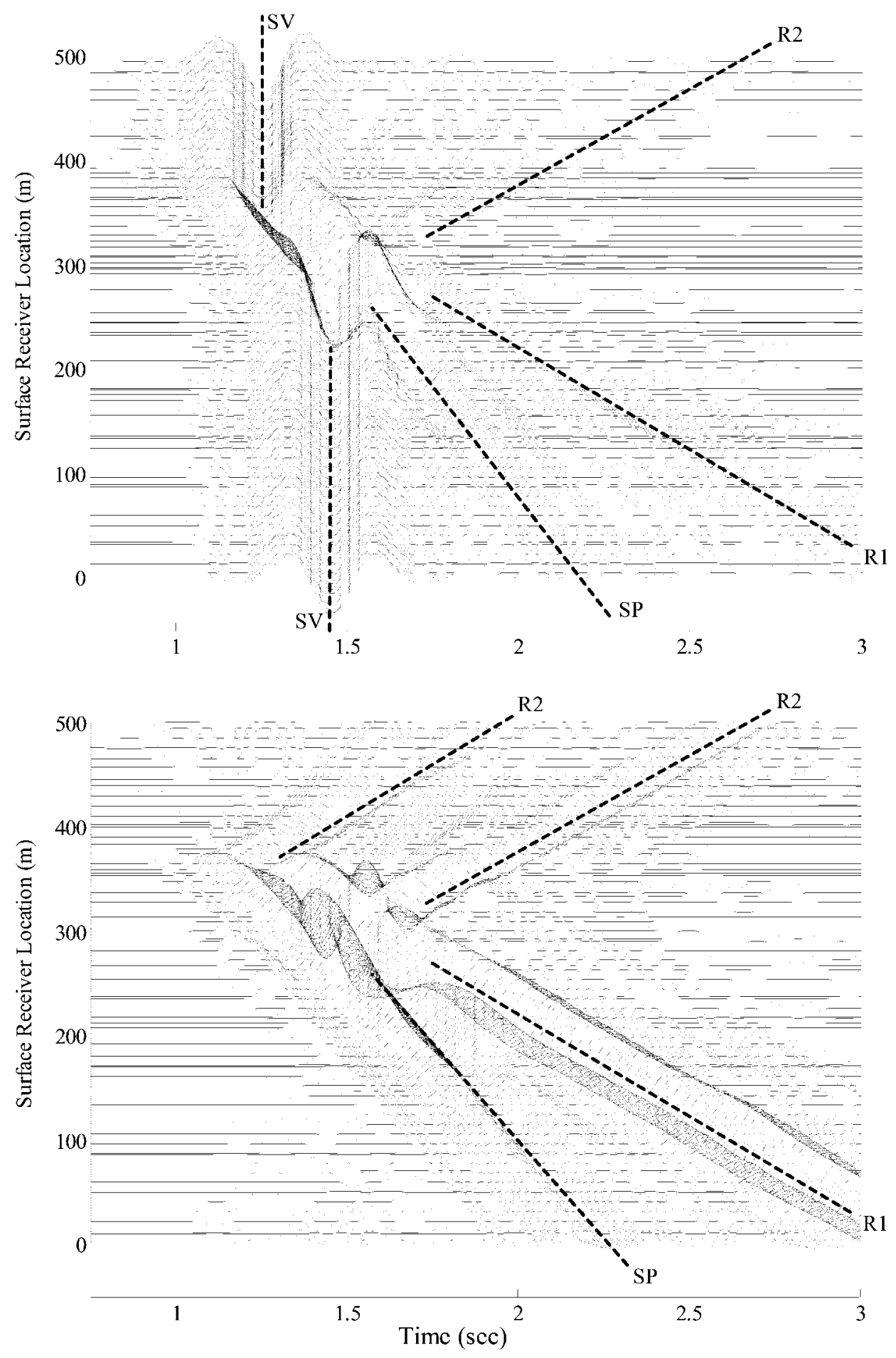

Figure 6. Synthetics of horizontal (top) and vertical (bottom) acceleration surface response for a cliff with $i=30^{\circ}$ slope subjected to vertically propagating $S V$ Ricker waves with $a_{0}=1.0$. Note that the crest is located at $x=300$.

diffraction potential, simply by considering the elastic response of the canyon cliff.

Observe also that although the particle motion of the incident seismic input is horizontally polarized (vertically propagating $S V$ waves), the surface response contains a parasitic vertical acceleration component as well. This corre- sponds to the vertical particle motion of surface-diffracted waves and is shown to carry a significant portion of the seismic wave energy.

Figure 7 illustrates the spatial distribution of peak horizontal and vertical acceleration, normalized by the far-field peak horizontal acceleration. For the illustrated example, 
namely a Ricker wavelet with central frequency $f_{0}=2.5 \mathrm{~Hz}$, the dominant wavelengths correspond roughly to the frequency interval $\left[0.5 f_{0}, 3 f_{0}\right]$, namely, $[0.67 \mathrm{H}, 4.16 \mathrm{H}]$, where $H$ is the height of the topographic irregularity. According to Bard (1999), topography effects are more pronounced for wavelengths comparable with the dimensions of the topographic feature. The narrow-band nature of the incident motion and the particular dimensions of the dominant wavelengths in this example emphasize the potential effects of topography: (1) the maximum aggravation of the primary (horizontal) acceleration component is approximately 50\%, and (2) the peak amplitude of the parasitic (vertical) component is on the order of $35 \%$ of the peak surface acceleration in the far field.

\section{Effect of Soil Stratigraphy}

We next identify the role of soil stratigraphy on the topographic aggravation of surface motion. We show that topography effects predicted for infinite wedges on the surface of homogeneous half-spaces are not representative of
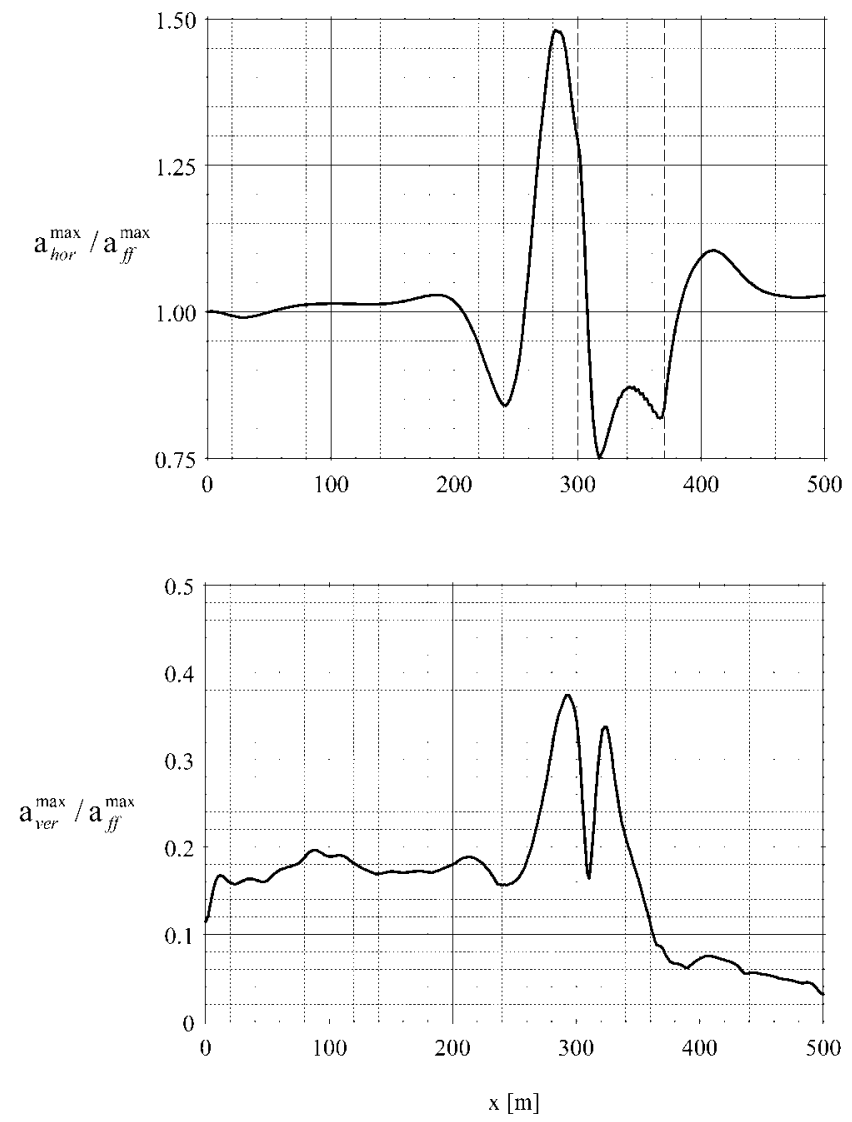

Figure 7. Spatial distribution of normalized horizontal (top) and vertical (bottom) peak acceleration along the ground surface for a cliff with $i=30^{\circ}$ slope subjected to vertically propagating $S V$ Ricker waves with $a_{0}=1.0$. Note that the crest is located at $x=$ 300 . clifftype topographies, when the response is also controlled by the soil profile characteristics.

For this purpose, we consider the idealized layered media schematically shown in Figure 8. We first simulate the response of a homogeneous layer overlying an elastic halfspace, followed by the response of a two-layered soil deposit. Both configurations are subjected to vertically incident Ricker wavelets with central frequency $f_{0}$, and the following dimensionless quantities are parametrically examined: (1) the dominant frequency of the input motion, normalized by the shear-wave velocity of the medium and the height of the topographic feature $a_{0}=2 f_{0}(H-h) / V_{s}$; (2) the bedrock-soil impedance ratio, $\rho_{R} V_{R} / \rho_{s} V_{s}$; (3) the depth-tobedrock ratio $h / H$; and (4) the relative thickness $\left(h_{1} /(H-\right.$ $h)$ ) and flexibility $\left(V_{s 1} / V_{s 2}\right)$ of a surface layer, for the twolayered soil profile (see Fig. 8b).

Our parametric simulations are outlined in Table 1. For all numerical analyses performed, material damping is selected to be $\xi=0.05(Q=10)$ for soil layers and $\xi=$ $0.01(Q=50)$ for the underlying bedrock, and Poisson's ratio is taken to be $v=0.35$ for both the soil layers and the underlying bedrock. For the parametric study, the density $\left(\rho_{R}\right)$ of the elastic half-space is chosen identical to that of the overlying soil deposit $\left(\rho_{s}\right)$ and therefore, the bedrocksoil impedance ratio is simply given by the ratio $\left(V_{R} / V_{s}\right)$.

(a)

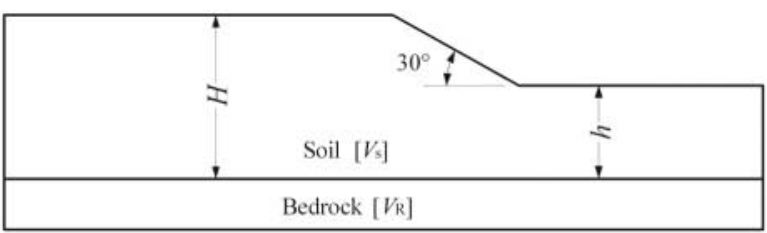

(b)

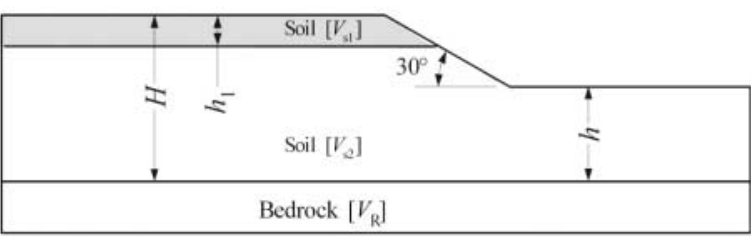

Figure 8. Schematic representation of the numerical models used in the parametric study.

Table 1

Dimensionless Variables Investigated to Identify the Effects of Soil Layering

\begin{tabular}{cccccc}
\hline \multicolumn{2}{c}{ Homogeneous Soil Layer } & & & \multicolumn{2}{c}{ Two-Layered Soil Profile } \\
$\begin{array}{c}a_{0}=2 f_{0} \\
(H-h) / V_{s}\end{array}$ & $\rho_{R} V_{R} / \rho_{s} V_{s}$ & $h / H$ & & $V_{s 1} / V_{s 2}$ & $h_{1} /(H-h)$ \\
\hline 0.5 & 1.0 & 0.25 & & 0.5 & 0.25 \\
1.0 & 2.0 & 0.50 & & 2.0 & 0.50 \\
1.5 & 10.0 & 0.75 & & \\
2.0 & & & & \\
\hline
\end{tabular}


Homogeneous Soil Layer. We first illustrate the frequencydependent nature of wave diffraction by surface topographic features, for a homogeneous half-space $\left(V_{R} / V_{s}=1.0 ; \mathrm{h} / \mathrm{H}\right.$ $=0.5$ ) subjected to vertically incident $S V$ Ricker wavelets with dimensionless frequencies $a_{0}=0.5,1.0,1.5$, and 2.0. Figure 9 shows the spatial distribution of peak surface acceleration (horizontal and vertical) behind the crest. For each simulation, the response is normalized by the peak acceleration at the far field, defined here at a distance $300 \mathrm{~m}$ from the vertex, where two-dimensional phenomena are shown to be negligible. Results are found to be in agreement with those reported by Ashford and Sitar (1997) and can be summarized as follows: (1) The location of peak horizontal acceleration behind the cliff is controlled by the dominant wavelength $\left(\lambda_{0}\right)$ of the incident motion (here, the central frequency of the Ricker wavelet) and is systematically observed at a distance $x_{\max }=0.2 \lambda_{0}$ from the crest. The amplitude of peak acceleration at this location is also frequency dependent
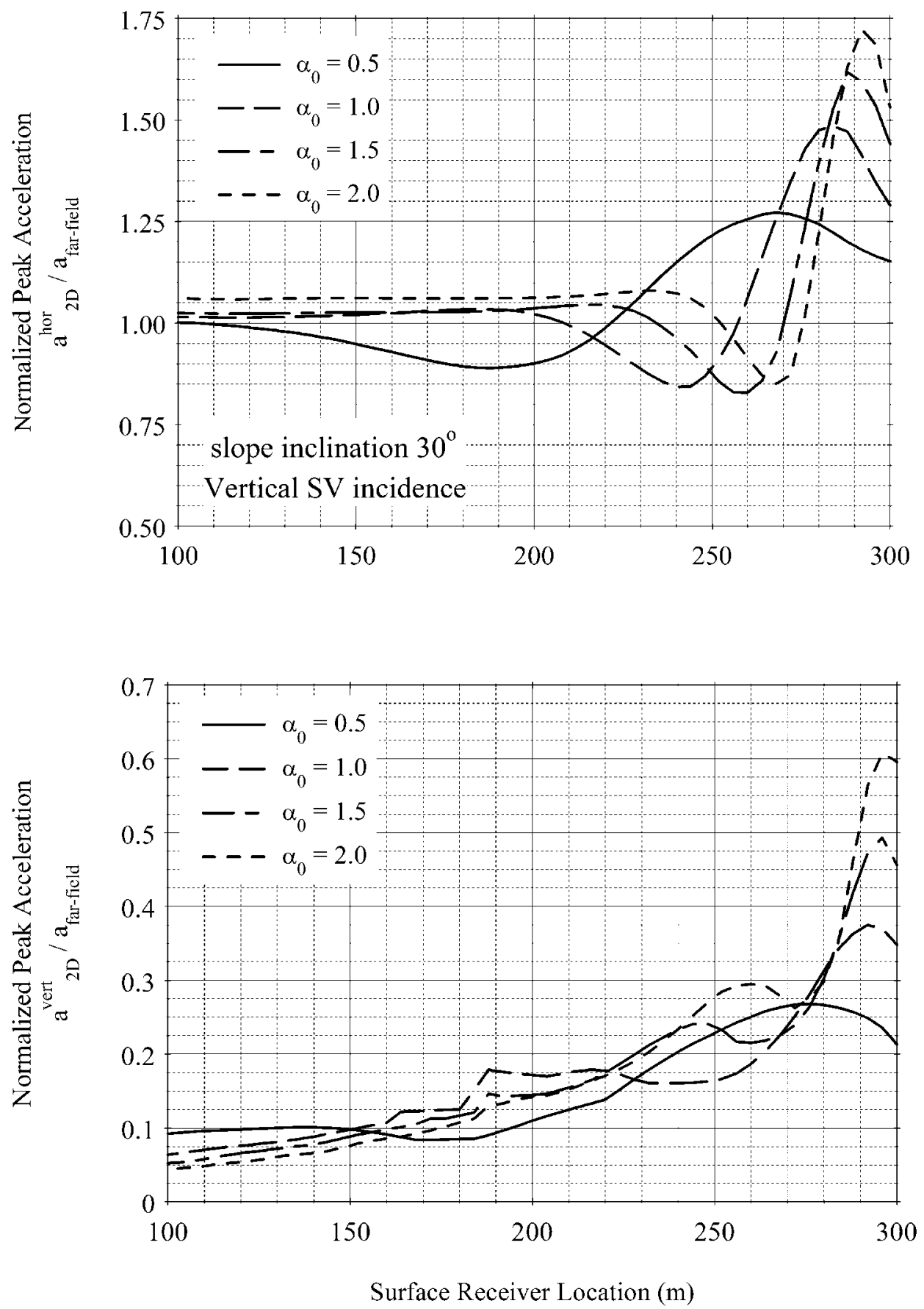

Figure 9. Normalized horizontal (top) and vertical (bottom) peak surface acceleration behind the crest as a function of the frequency content of the incident pulse, for $V_{R} / V_{s}=1.00$ and $h / H=0.50$. Note that the crest is located at $x=300$. 
and increases almost linearly with frequency. (2) The amplitude of the parasitic acceleration component is also frequency dependent. For the high-frequency input pulse in these simulations, the peak vertical response is on the order of $60 \%$ of the corresponding far-field response. Because the location of peak vertical acceleration is also frequency controlled, higher-frequency components are amplified within a narrower zone in the vicinity of the crest.

Numerical simulations are next performed for a homogeneous layer overlying an elastic half-space, for various depth-to-bedrock ratios $(h / H)$ and bedrock-soil impedance contrasts $\left(V_{R} / V_{s}\right)$. Our results show that the bedrock-soil impedance, which controls the seismic energy trapped in the surface layer and the corresponding one-dimensional amplification of incident motion, introduces additional complexity to the problem studied. In particular, (1) resonance phenomena associated with the response of the one-dimensional soil column in front of the slope toe (shallow profile) result in consecutive reflections of the seismic energy and continuous generation of surface waves at the lower corner of the cliff. These waves travel toward the crest and enhance significantly the forward-scattered surface waves that are generated on incidence of primary $S V$ waves on the slope. (2) Resonance phenomena associated with the one-dimensional soil configuration behind the crest (deep profile) alter the constructive interference mechanism of diffracted and excessively amplified incident waves close to the vertex and result in additional aggravation of the surface motion.

Figure 10 shows contour plots of the Fourier amplitude of horizontal surface acceleration, for depth-to-bedrock ratio $h / H=0.25$ and the various bedrock-soil impedances examined. Soil stratigraphy clearly controls not only the absolute intensity of surface motion, but also the frequency content and spatial distribution of the response. Constructive and destructive interference of incident and diffracted waves along the surface results in significant differential motion, both behind the crest and along the slope, where transition occurs between the convex and concave part of the topography.

Figures $11 \mathrm{a}$ and $11 \mathrm{~b}$ plot the spatial distribution of normalized horizontal and vertical acceleration, respectively, for the slope geometry of the Kifissos canyon and for bedrock-soil impedance $V_{R} / V_{s}=10.00$. Results are shown for all depth-to-bedrock ratios $(h / H)$ and all dimensionless frequencies $\left(a_{0}\right)$ examined. The spatial distribution of vertical acceleration, which arises purely from diffraction of incident waves and is enhanced by the surface waves generated along the crest, is particularly sensitive to the stratigraphy of the far field.

To identify the effects of far-field resonance on the topographic aggravation mechanism, the fundamental frequency of the shallow $\left(f_{1}^{h}\right)$ and deep $\left(f_{1}^{H}\right)$ far-field soil columns, is normalized by the central frequency of the incident motion $\left(f_{0}\right)$ in Table 2 , for each of the aforementioned analyses; the cases in which resonance of either far-field profile is observed are highlighted on the same table. Also, cumu-
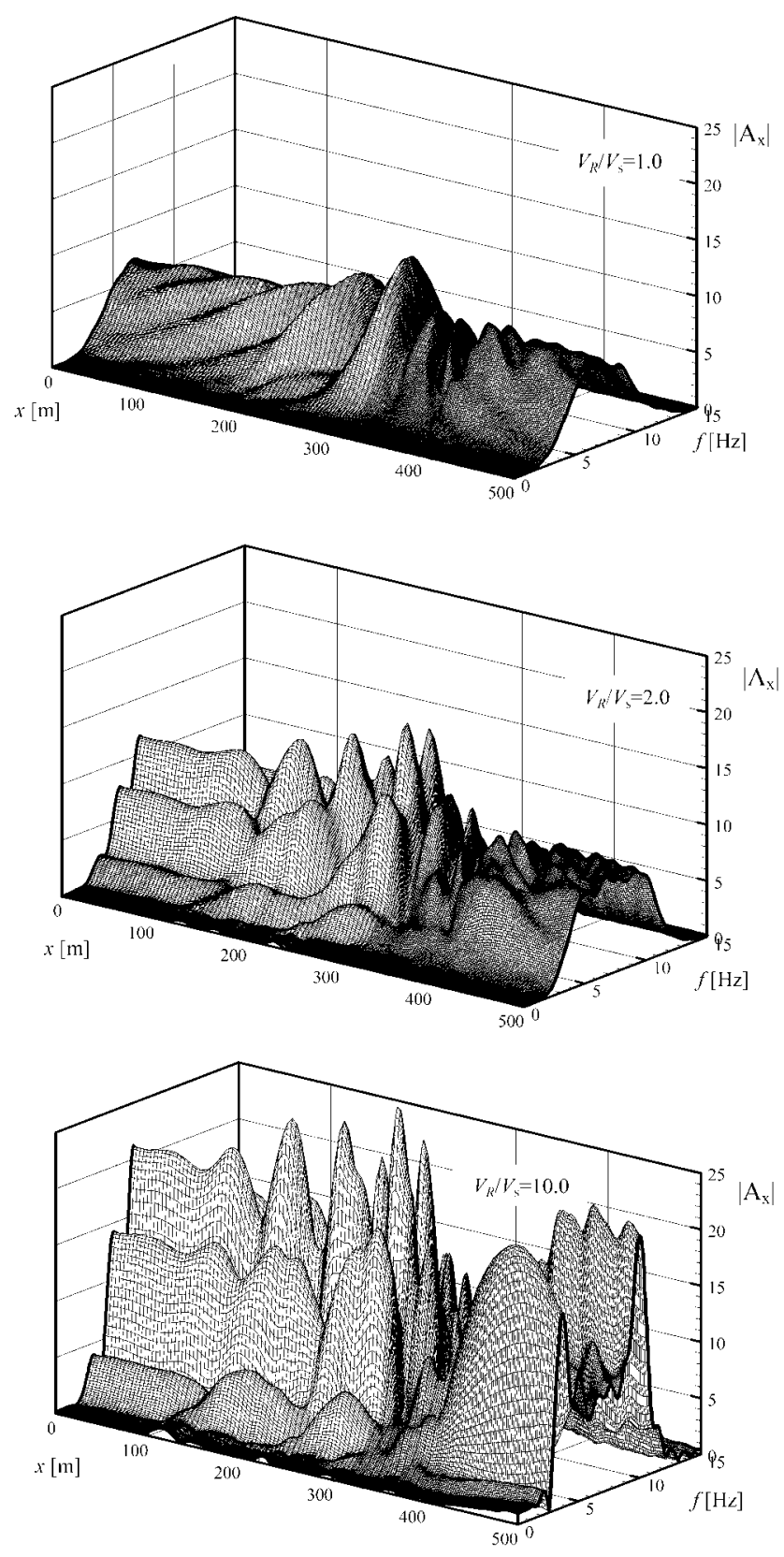

Figure 10. Fourier amplitude contours of surface horizontal acceleration as a function of the bedrocksoil impedance ratio, for $h / H=0.25$ and $a_{0}=2.0$.

lative results for the peak-normalized horizontal and vertical acceleration behind the crest as a function of dimensionless frequency $a_{0}$ are shown in Figure 12. The cases in which resonance of either far-field soil columns occurs and alters the topographic aggravation mechanism are also identified. Finally, seismogram synthetics of the horizontal and vertical surface acceleration are illustrated in Figures 13a and 13b for the configuration with depth-to-bedrock ratio $h / H=$ 0.25 , where resonance of the shallow and the deep far field is observed for $a_{0}=1.5$ and $a_{0}=0.5$, respectively.

The governing role of soil profile can be readily ob- 
(a)
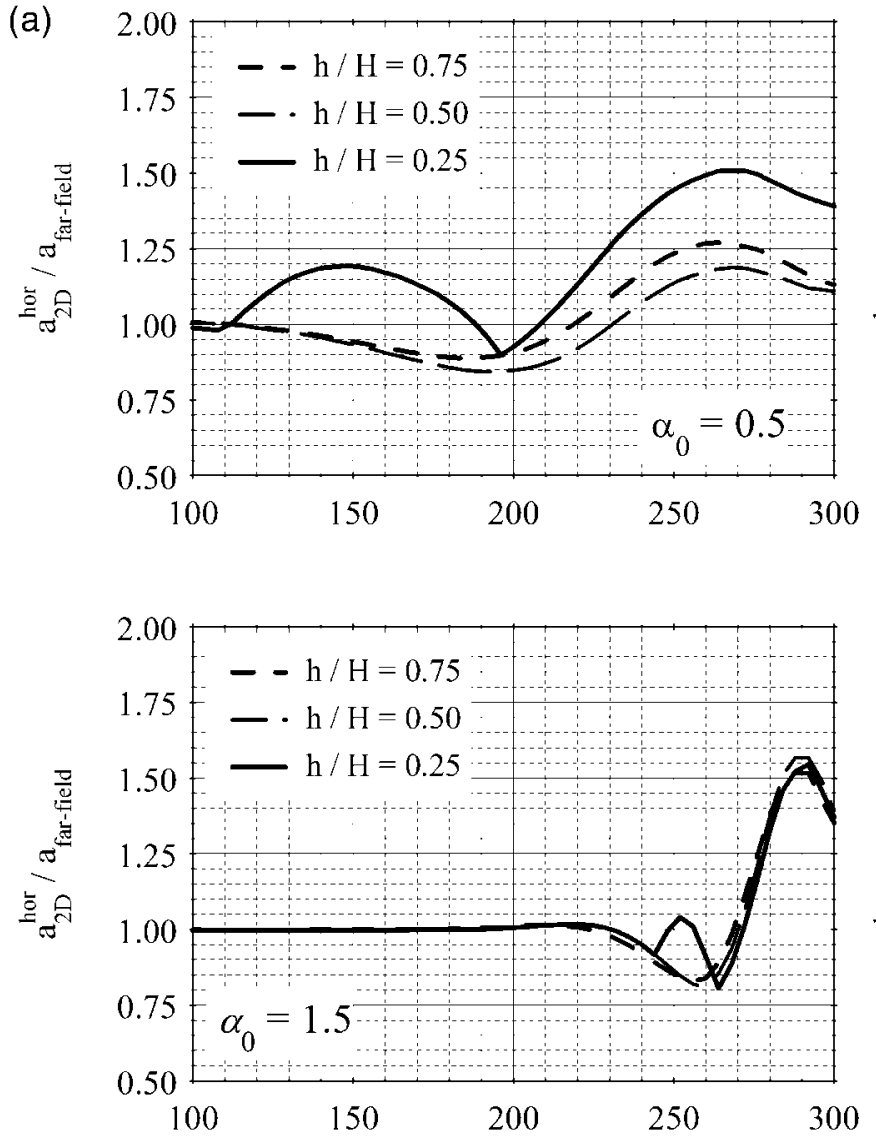

Surface Receiver Location (m)
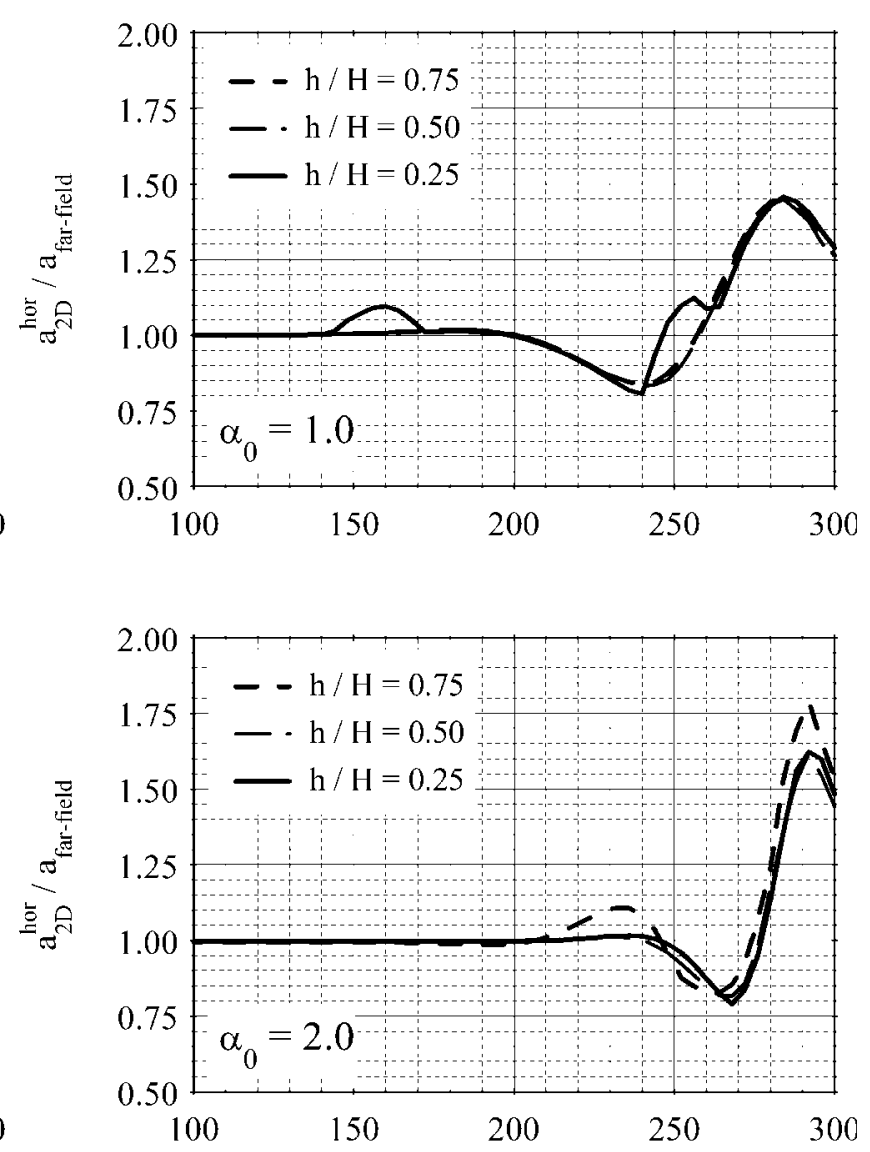

Surface Receiver Location (m)

Figure 11. (a) Effect of depth-to-bedrock ratio $(h / H)$ on the spatial distribution of normalized peak horizontal surface acceleration $\left(V_{R} / V_{s}=10.00\right)$. (b) Effect of depthto-bedrock ratio $(h / H)$ on the spatial distribution of normalized peak vertical surface acceleration $\left(V_{R} / V_{s}=10.00\right)$.

(continued)

served in the foregoing figures. In particular, upon resonance of the shallow far-field soil column (Fig. 13a), the vertical response close to the vertex is shown to be significantly enhanced. Note that this parasitic component evolves from wave diffraction at the cliff toe and excess amplification of the motion at the shallow far field and results in continuous generation of high-amplitude surface waves that travel along the slope toward the crest. In addition, the frequency characteristics of the horizontal response are altered with components that correspond to the resonant frequencies of the shallow column that are amplified in a broad zone behind the crest. Nonetheless, the magnitude and spatial distribution of the normalized peak horizontal acceleration along the surface behind the crest are not significantly affected, when compared with the ensemble of simulations in which no resonance phenomena are encountered.

Upon resonance of the deep far field (Fig. 13b), the spatial distribution and magnitude of the peak-normalized horizontal acceleration behind the crest is affected, because of altering of constructive interference mechanisms. The resulting two-dimensional aggravation of motion is more in- tense $(50 \%$ as opposed to $25 \%$ predicted for $h / H=0.50$ and 0.75 ) and occurs in a broader zone behind the crest. By contrast, the frequency content of the primary response and the amplitude of the parasitic acceleration component (which is governed by the response of the shallow column) are practically unaffected.

Two-Layered Soil Profile. We here examine the effects of a shallow soil layer on the topographic aggravation of motion as a function of its relative thickness and impedance contrast to the underlying soil deposit (Fig. 8b). In particular, we illustrate the effects of soil layering on the wave diffraction and constructing interference mechanism, which occurs in the vicinity of the crest and results in excessive amplification of the incident motion. We show that the altering of these phenomena because of multiple reflections and refractions, which occur within the surface layer and at the soillayer interface, respectively, results in enhancement or deduction of the topographic aggravation predicted for a homogeneous soil layer in the preceding section.

The spatial variation of normalized horizontal and ver- 

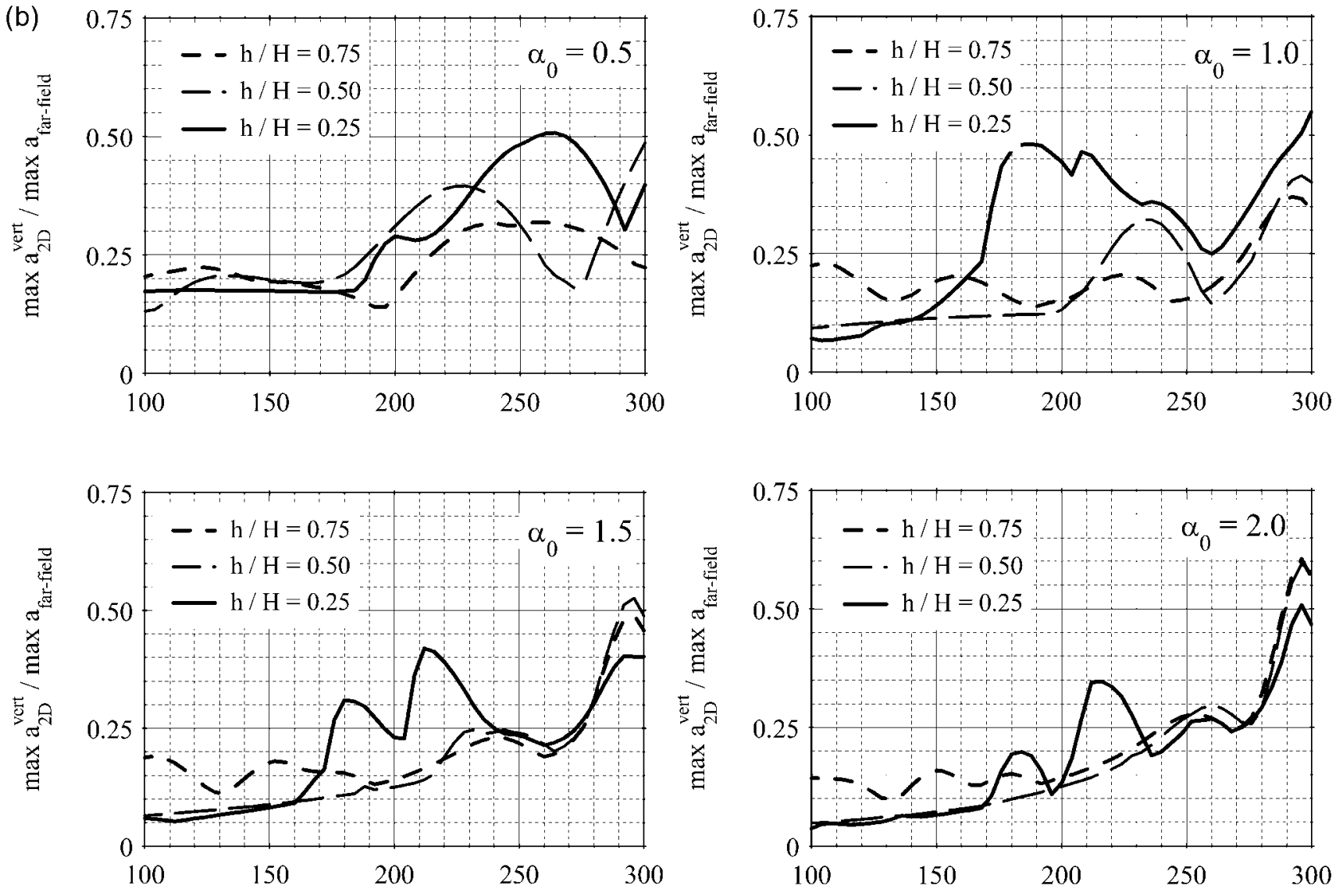

Surface Receiver Location (m)

Surface Receiver Location (m)

Figure 11. Continued.

Table 2

Ratio of the Fundamental Frequencies of the Shallow and Deep Far-Field Soil Columns to the Central Frequency of the Incident Ricker Pulse

\begin{tabular}{|c|c|c|c|c|}
\hline \multirow{2}{*}{\multicolumn{2}{|c|}{$f_{1}^{h} / f_{0}$}} & \multicolumn{3}{|c|}{$h / H$} \\
\hline & & 0.25 & 0.5 & 0.75 \\
\hline \multicolumn{5}{|c|}{ Shallow far-field soil columns } \\
\hline \multirow{4}{*}{$a_{0}$} & 0.5 & 2.86 & 1.00 & 0.33 \\
\hline & 1.0 & 1.43 & 0.50 & 0.17 \\
\hline & 1.5 & 0.95 & 0.33 & 0.11 \\
\hline & 2.0 & 0.71 & 0.25 & 0.08 \\
\hline \multicolumn{5}{|c|}{ Deep far-field soil columns } \\
\hline \multirow[t]{4}{*}{$a_{0}$} & 0.5 & 0.93 & 0.50 & 0.25 \\
\hline & 1.0 & 0.37 & 0.25 & 0.13 \\
\hline & 1.5 & 0.25 & 0.17 & 0.08 \\
\hline & 2.0 & 0.19 & 0.13 & 0.06 \\
\hline
\end{tabular}

tical acceleration behind the crest is shown in Figures $14 \mathrm{a}$ and $14 \mathrm{~b}$, for a surface layer with relative thickness $h_{1} /(H-$ $h)=0.25$ overlying a homogeneous half-space $\left(V_{R} / V_{s 2}=\right.$ 1.0). Results are shown for all values of soil-to-soil impedance contrast $\left(V_{s 1} / V_{s 2}\right)$ and all dimensionless frequencies $\left(a_{0}\right)$ investigated, whereas the slope inclination of the canyon $\left(i=30^{\circ}\right)$ and the depth-to-bedrock ratio $(h / H=0.50)$ are kept constant for all simulations. Note that the case of $V_{s 1} /$ $V_{s 2}=1.0$, which is also plotted, corresponds to the homogeneous half-space case shown in Figure 9. Figure 15 successively illustrates snapshots of the acceleration wave field and contours of the absolute acceleration amplitude for a soft $\left(V_{s 1} / V_{s 2}=0.5\right)$ and a stiff $\left(V_{s 1} / V_{s 2}=2.0\right)$ surface-layer configuration, subjected to vertically propagating $S V$ Ricker wavelets with dimensionless frequency $a_{0}=2.0$. For this case, Figure 16 plots the Fourier amplitude contours of surface horizontal acceleration as a function of the soil-layers impedance ratio. Results are compared with the frequency content of the response on the surface of a homogeneous half-space (see also Fig. 10a).

The main results of our investigation can be summarized as follows. (1) In the soft-surface-layer case $\left(V_{s 1} / V_{s 2}\right.$ $=0.5)$, the incident wave energy is trapped within the surface layer, and multiple reflections interact with the surface waves that originate from the lower corner of the slope and propagate uphill. The scattered wave field is composed here of Rayleigh waves, which are generated at the crest and travel along the surface with the Rayleigh wave velocity of 

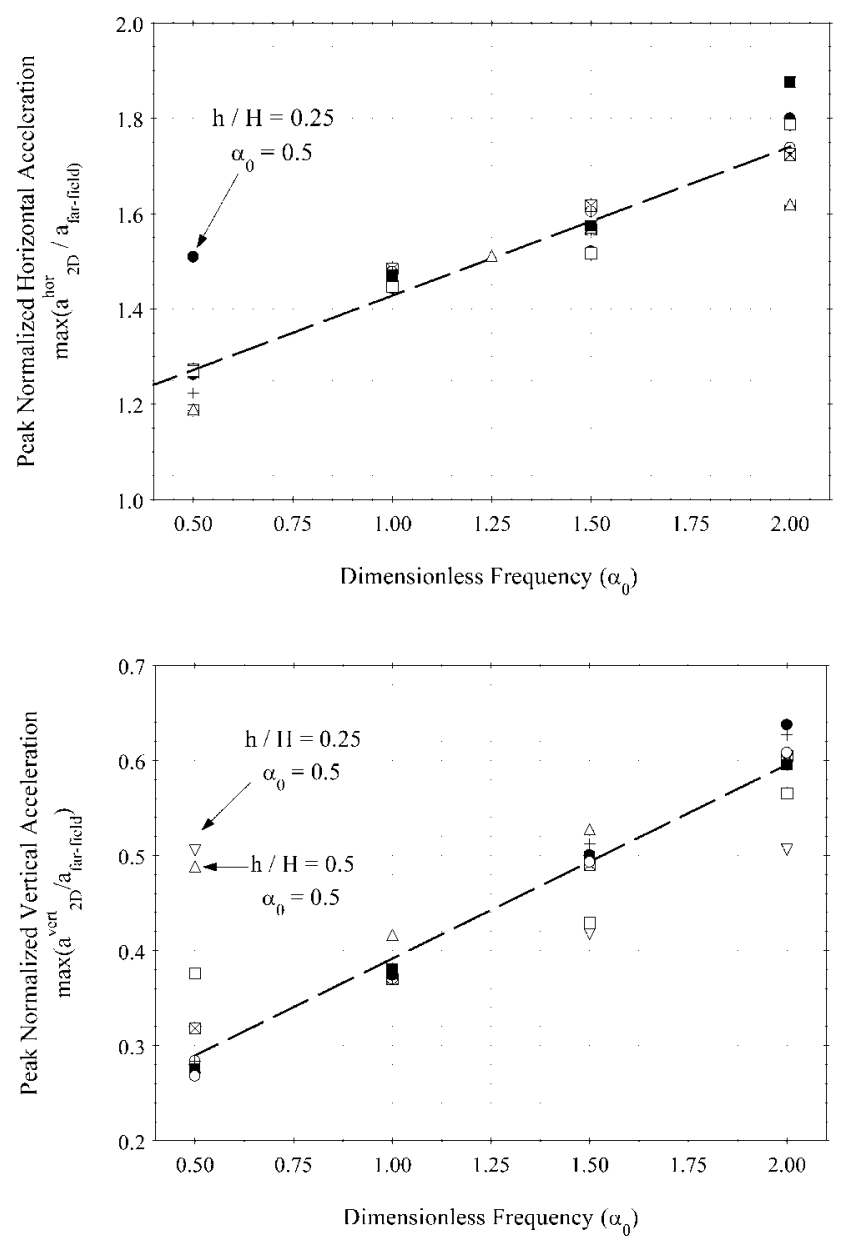

Figure 12. Cumulative results of the peak normalized horizontal and vertical surface acceleration, as a function of the dimensionless frequency $\left(a_{0}\right)$ for the ensemble of our parametric simulations.

the surface layer, $V_{\mathrm{R} 1}$, and reflections of waves that travel along the soil-layers interface with the Rayleigh wave velocity of the lower layer, $V_{\mathrm{R} 2}$ (see Fig. 15). (2) In the hardlayer case $\left(V_{s 1} / V_{s 2}=2.0\right)$, the incident energy is almost completely reflected at the soil-layers interface. The corresponding seismogram synthetics of surface horizontal acceleration only imprint incident $S V$ waves, whereas the vertical component consists of a single reflection that corresponds to Rayleigh waves, originated at the toe of the cliff (see Fig. 15). More specifically for the soft-surfacelayer case, (3) for relatively high-frequency incident waves, namely wavelengths on the order of magnitude of the surface-layer thickness, the peak-normalized horizontal acceleration of the stratified medium is lower than the corresponding acceleration of the homogeneous half-space. Note, however, that the absolute motion amplification is very significant in the soft-surface-layer case, a fact that illustrates the dominant role of the far-field stratigraphy on the amplification mechanism behind the crest (see Figs. 14a and 16). (4) The vertical acceleration component is remarkably en- hanced. This effect is prominent for incident waves with wavelengths short enough to see the surface layer, that is, for input signals rich in high frequencies. In this case, the vertical acceleration is shown to attain amplitudes $25 \%$ larger than the corresponding response at the far field. (5) In the frequency domain, the far-field/two-dimensional transfer function is very erratic for wavelengths comparable with the thickness of the surface layer, that is, its resonant frequencies.

By means of the foregoing simulations, we have shown that soil stratigraphy not only controls the far-field amplification or deamplification of motion, but also plays a major role in the aggravation mechanism by irregular topographic features. In particular, for the soft-surface layer examined, which is not only an idealized model for typical soil profiles in Adàmes but also for many sedimentary soil deposits, the vertical component of acceleration is found to be excessively amplified; this alone could explain the extensive structural damage behind the vertex that was observed during the Athens 1999 event.

\section{Effect of Soil Heterogeneity}

In classical seismology, seismic attenuation is usually considered to be a combination of two mechanisms, so-called intrinsic absorption and scattering loss. Intrinsic absorption refers to the conversion of vibration energy into heat and results in attenuation of seismic wave amplitude with travel distance. In our numerical simulations, this mechanism is approximated by means of hysteretic (rate-independent) material damping (i.e., frequency-independent $Q$ ).

On the other hand, scattering occurs as seismic waves propagate though a medium with randomly distributed heterogeneities that exhibit multiple sizes and contrasts in physical properties. Because of energy redistribution, seismic wave amplitude appears also to decay with travel distance (Aki, 1980), a fact that renders scattering a mechanism of phenomenological attenuation. It has been also shown (Aki, 1980; Sato, 1982; Wu, 1982a; Sato and Fehler, 1998) that the characteristic scale of the heterogeneity having the most influence on a given wave is usually of the same order of magnitude as the dominant wavelengths of the seismic wave.

To assess the effects of soil heterogeneity, we use the geometry characteristics of the Kifissos river canyon and simulate the soil deposit as a random medium; next, we illustrate the net effects of simultaneous scattering by material heterogeneity and irregular surface topography, as a function of the correlation distance normalized by the dominant propagating wavelengths.

For this purpose, we generate univariate Gaussian stochastic fields, using separable correlation structures to describe the spatial distribution of shear-wave velocity in the horizontal and vertical direction. We successively employ the Monte-Carlo technique by mapping the stochastic prop- 

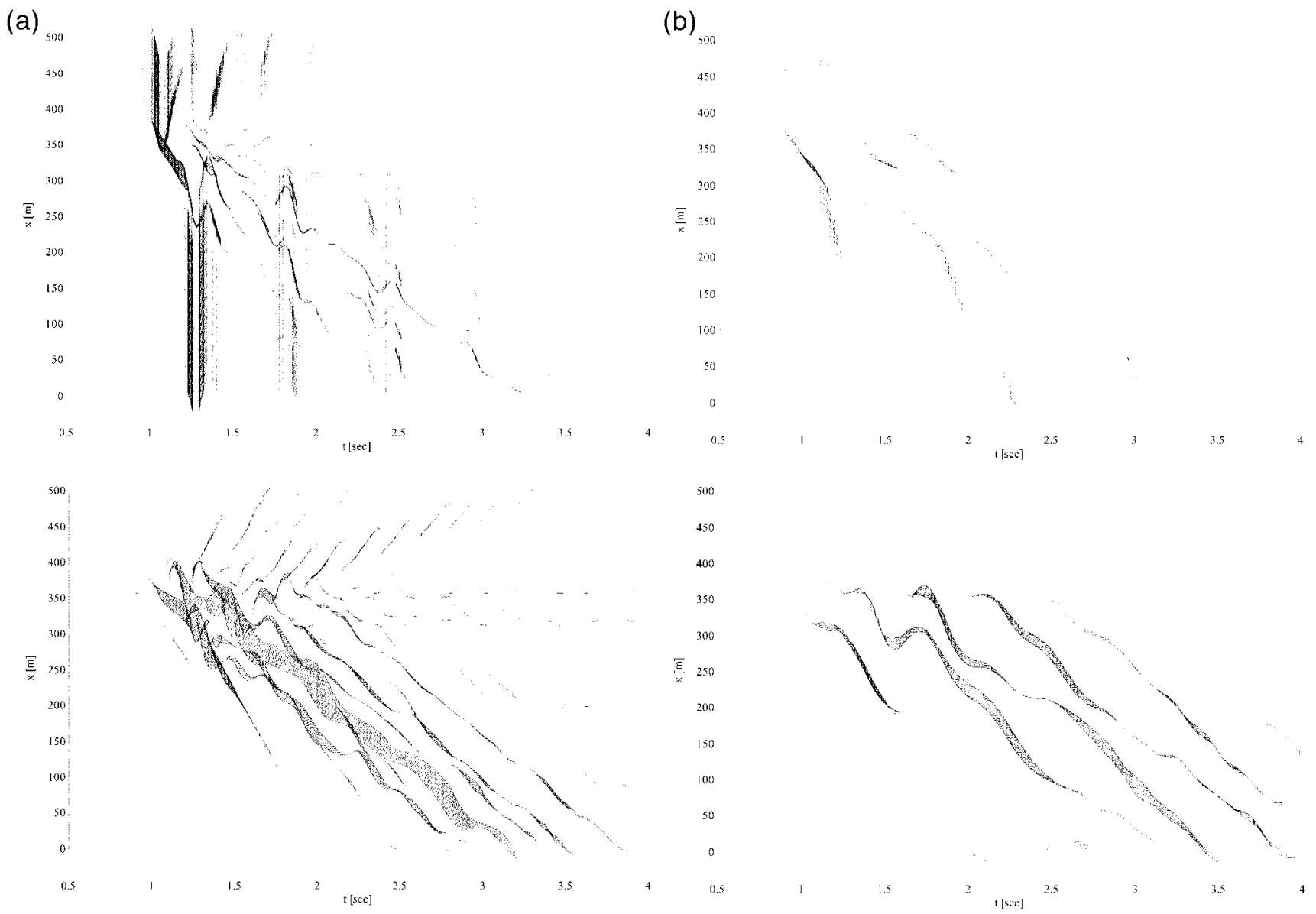

Figure 13. (a) Seismogram synthetics of the horizontal and vertical surface acceleration, for the case: $h / H=0.25, a_{0}=1.5$ (resonance of the shallow far-field soil column). (b) Seismogram synthetics of the horizontal and vertical surface acceleration, for the case: $h / H=0.25, a_{0}=0.5$ (resonance of the deep far-field soil column).

erties on the finite-element model and perform deterministic simulations.

Stochastic Description of Soil Properties. The necessarily limited number of soil tests and the difficulties associated with reproducing in situ material behavior are significant sources of uncertainty in the evaluation of site-response analyses, whereas the uncertainty about the accuracy of analytical or numerical models used for the analysis has been shown, in general, to be less significant. Moreover, the uncertainty in field data is increased by the inherent soil heterogeneity, namely, spatial variability of soil properties within so-called homogeneous soil layers. As a result, deterministic descriptions of the spatial variability of soil properties are not always feasible, and the sufficiently large degree of disorder exhibited leads to the use of statistical methods to describe their distribution within "statistically homogeneous" soil zones.

Results of numerous studies on the probability distribution of soil properties are reported in the literature. Based on field measurements and empirical correlations, research- ers have fitted both Gaussian and non-Gaussian stochastic fields to various soil conditions (Schultze, 1971). According to Popescu (1995), who studied the effects of spatial variability of soil properties on the liquefaction potential of soil deposits: First, most soil properties exhibit skewed nonGaussian distributions. The Gaussian correlation function characterizes single-scale smoothly heterogeneous media, whereas real heterogeneities in the earth are often multiscaled. Flatté and Wu (1988) also showed that the exponential or Kolmogorov correlation functions fit the data much better than the Gaussian correlation function. The nonGaussian nature of the lithospheric heterogeneities has been established from velocity well-logging data as well (Sato, 1979; Wu, 1982). Second, each soil property can follow different probability distributions for various materials and sites; therefore, the statistics and the shape of the distribution function have to be estimated for each case. In this respect, based on a limited amount of field data, Popescu et al. (1998a) have shown that the soil strength is likely to follow skewed probability distributions for shallow layers and more symmetrical ones for deep layers. 

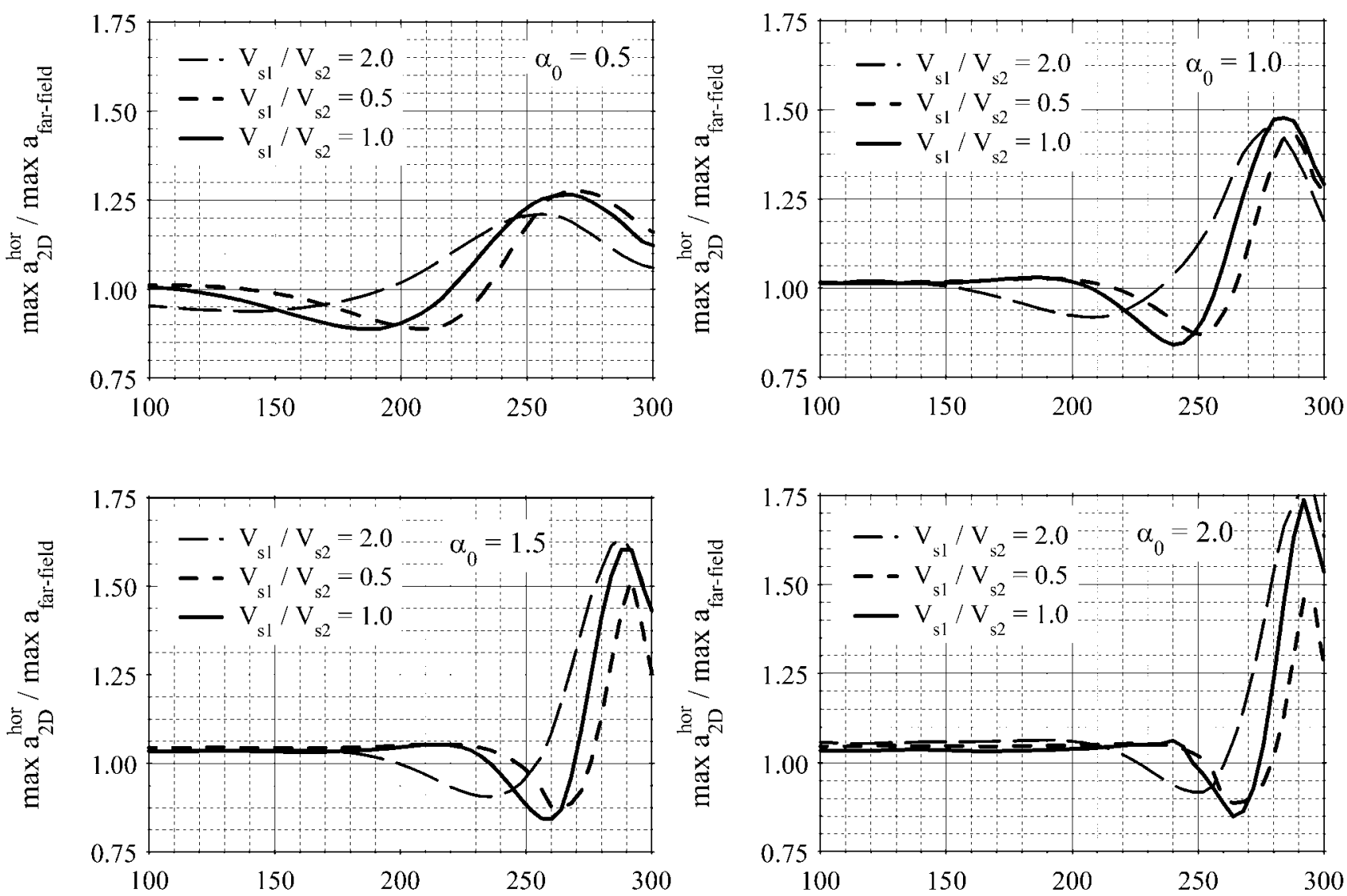

Surface Receiver Location (m)

Surface Receiver Location (m)

Figure 14. (a) Effect of the stiffness of the surface layer $\left(V_{s 1} / V_{s 2}\right)$ on the spatial distribution of normalized peak horizontal surface acceleration $\left(V_{R} / V_{s 2}=1.0\right)$. (b) Effect of the stiffness of the surface layer $\left(V_{s 1} / V_{s 2}\right)$ on the spatial distribution of normalized peak vertical surface acceleration $\left(V_{R} / V_{s 2}=1.0\right)$.

(continued)

Once the probabilistic characteristics of the spatial variation of soil properties are known, sample functions of the underlying stochastic field can be digitally simulated using one of the methods referred in the literature, such as the autoregressive moving-average (ARMA) models, the covariance matrix decomposition method, the spectral representation method, and the conditional probability function method (for further details, see Soong and Grigoriu [1993]).

Nonetheless, non-Gaussian distributions require knowledge of moments of all orders, unlike Gaussian fields where the first two moments provide complete probabilistic information. This renders the estimation procedure cumbersome, because it is difficult to estimate moments higher than order two from actual (non-Gaussian) data. Therefore, the simulation of material properties is done in practice by using limited available probabilistic information, namely the cross-spectral density matrix and the marginal probability distribution functions.

Because the selection of Gaussian probability functions allows a more controlled parametric study, especially when no flexibility of the distribution function is necessary, as would be the case for curve-fitting purposes to a potential empirical distribution function, here we generate Gaussian shear-wave velocity stochastic fields using the exponential decaying spectral density function (SDF):

$$
\rho_{i}(\xi)=\cos \left[2 \tan ^{-1}\left(\frac{\xi}{\theta_{i}}\right)\right] /\left[1+\left(\frac{\xi}{\theta_{i}}\right)^{2}\right]
$$

where $\rho_{i}$ is the correlation function in spatial direction $i$ (horizontal or vertical), $\xi_{i}$ is the separation distance, and $\theta_{i}$ is the correlation distance in the $i$ th direction. Separate correlation structures are assigned to the horizontal and vertical direction to account for the mechanisms of soil deposit formation (Vanmarcke, 1983). The correlation function of the stochastic field is defined as their product. The random fields are generated in the wavenumber domain by using the spectral representation method (Shinozuka and Deodatis, 1996) and then denormalized by using a depth-independent standard deviation $\sigma=0.15 V_{s \text {,mean }}$, where $V_{s, \text { mean }}$ is the shear-wave velocity of the background medium; the random fields are then mapped on deterministic finite-element models. 

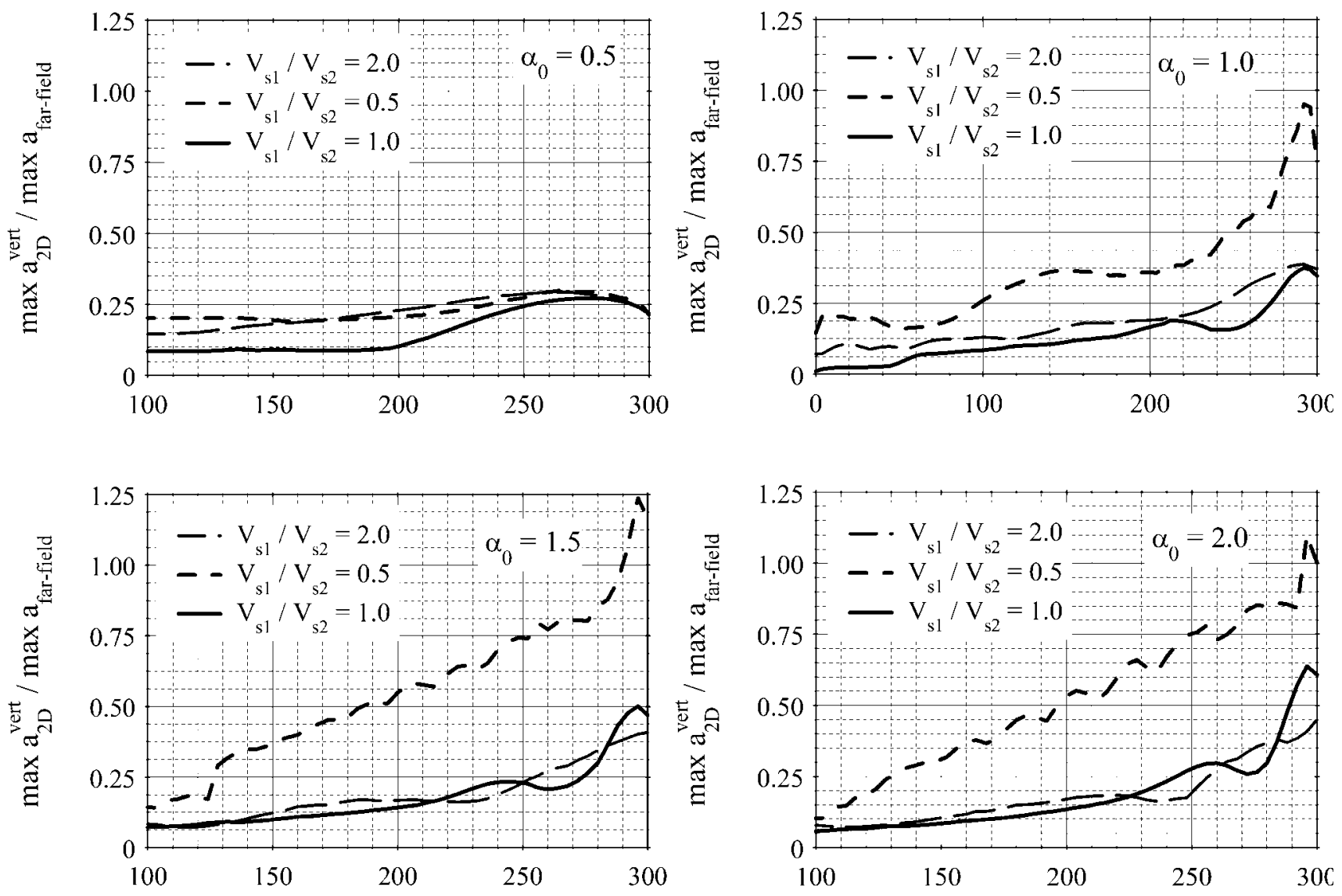

Surface Receiver Location (m)

Surface Receiver Location (m)

Figure 14. Continued.

Table 3 outlines our parametric simulations for the vertical correlation distance that was estimated for typical soil profiles in Adàmes, namely $\theta_{z}=2.5 \mathrm{~m}$. Note that for all the analyses performed, the vertical correlation distance is selected to be smaller than the corresponding value in the horizontal direction, a typical characteristic of sedimentary soil deposits. Figure 17 shows a three-dimensional illustration of the SDF for typical simulated random fields, along with realizations of the corresponding random medium mapped on the deterministic finite-element mesh.

Deterministic Finite-Element Analyses. In our investigation, we use the Monte Carlo simulation method by combining digital simulations of univariate (shear-wave velocity), multidimensional (horizontal and vertical direction), and Gaussian stochastic fields with deterministic finiteelement analyses. The vector field is first expressed in terms of the elastic soil stiffness, and a mapping technique is next used to imprint the sample fields on the finite-element mesh. For this purpose, we use the so-called midpoint method, where the random field is represented by its values at the centroids of the finite elements. In summary, the method involves three steps:
1. Digital generation of sample functions of a two-dimensional, Gaussian stochastic field, where each simulated sample function represents a possible realization of shearwave velocity over the discretized domain.

2. Mapping of the random variables at the finite-element centroids.

3. Deterministic finite-element analyses for each shearwave velocity sample field.

Obviously, a sufficient number of finite-element simulations are necessary to establish the statistics of the response. In this study, we performed 40 realizations for each stochastic field, a number primarily restricted by the computational effort that is associated with each simulation. Nonetheless, it is considered sufficient to capture the fundamental effects of soil randomness both on the time- and frequency-domain characteristics of the surface response.

The incident motion in the numerical simulations consists of Ricker wavelets with varying central frequency $f_{0}$. Time-domain results are here illustrated for a single Ricker pulse with central frequency $f_{0}=5.0 \mathrm{~Hz}$, and frequencydomain results are illustrated for a train of three Ricker wavelets with $f_{0}=3.0 \mathrm{~Hz}, 5.0 \mathrm{~Hz}$, and $10.0 \mathrm{~Hz}$, respec- 

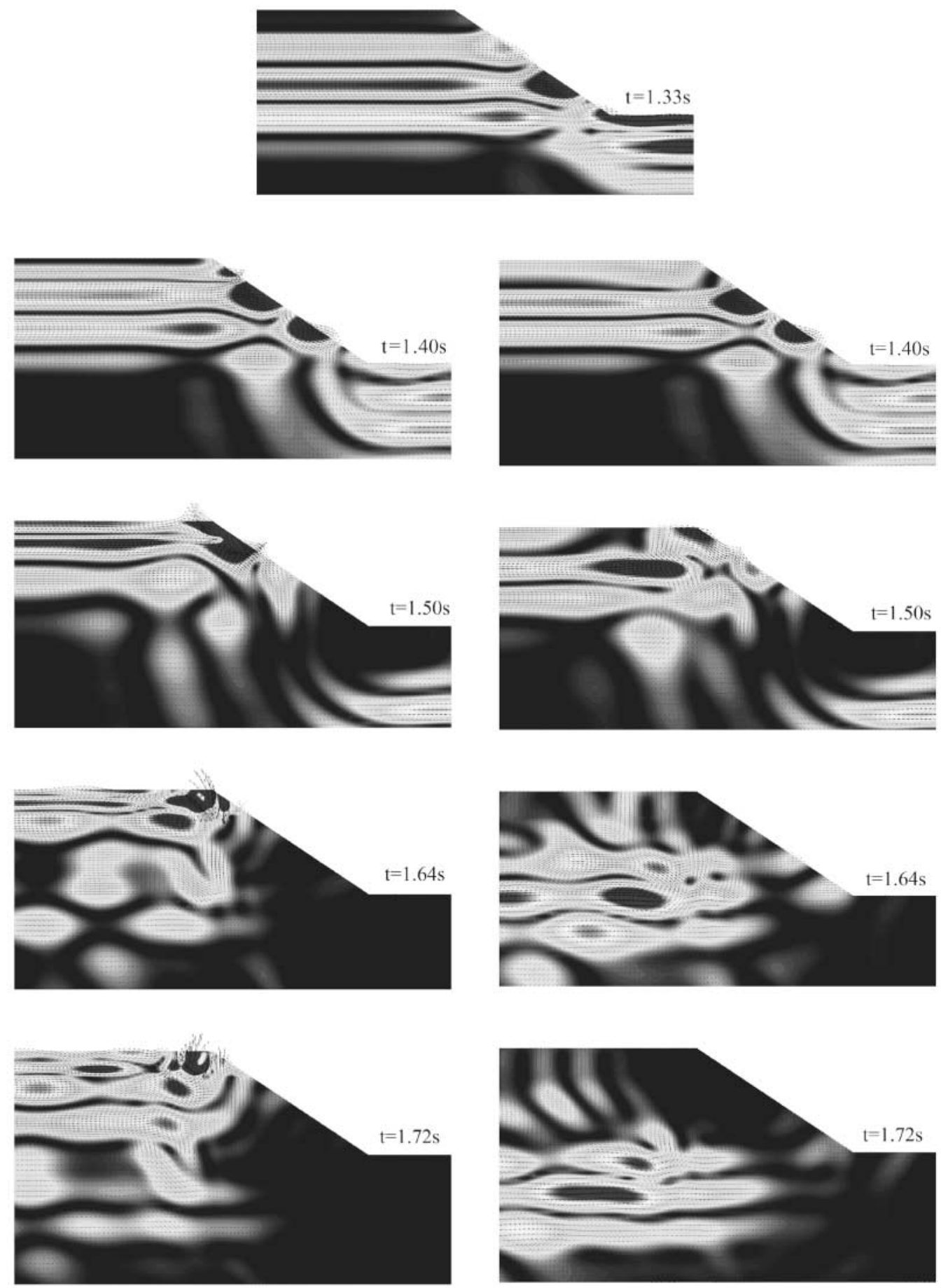

Figure 15. Snapshots of acceleration wave field and contours of absolute acceleration amplitude for a two-layered soil configuration with $h_{1} /(H-h)=0.25, V_{R} / V_{s 2}$ $=1.0, h / H=0.5$. Soft layer case, $V_{s 1} / V_{s 2}=0.5$ (left); stiff layer case, $V_{s 1} / V_{s 2}=2.0$ (right).

tively. For each case, results are compared with the response of a homogeneous medium with the background shear-wave velocity of the random field.

Figure 18 shows the mean and standard deviation of the spatial distribution of normalized peak surface acceleration for the ensemble of simulations that correspond to the sto- chastic fields illustrated in Figure 17, namely $\theta_{z}=2.5 \mathrm{~m}$ and $\theta_{x}=5.0 \mathrm{~m}, 25.0 \mathrm{~m}$, and $100.0 \mathrm{~m}$. In the frequency domain, Figure 19 illustrates the mean distribution of the crest-to-far-field and crest-to-base transfer functions for all the correlation distances $\theta_{x}$ investigated. Finally, Figure 20 plots Fourier amplitude surfaces of the ground horizontal 

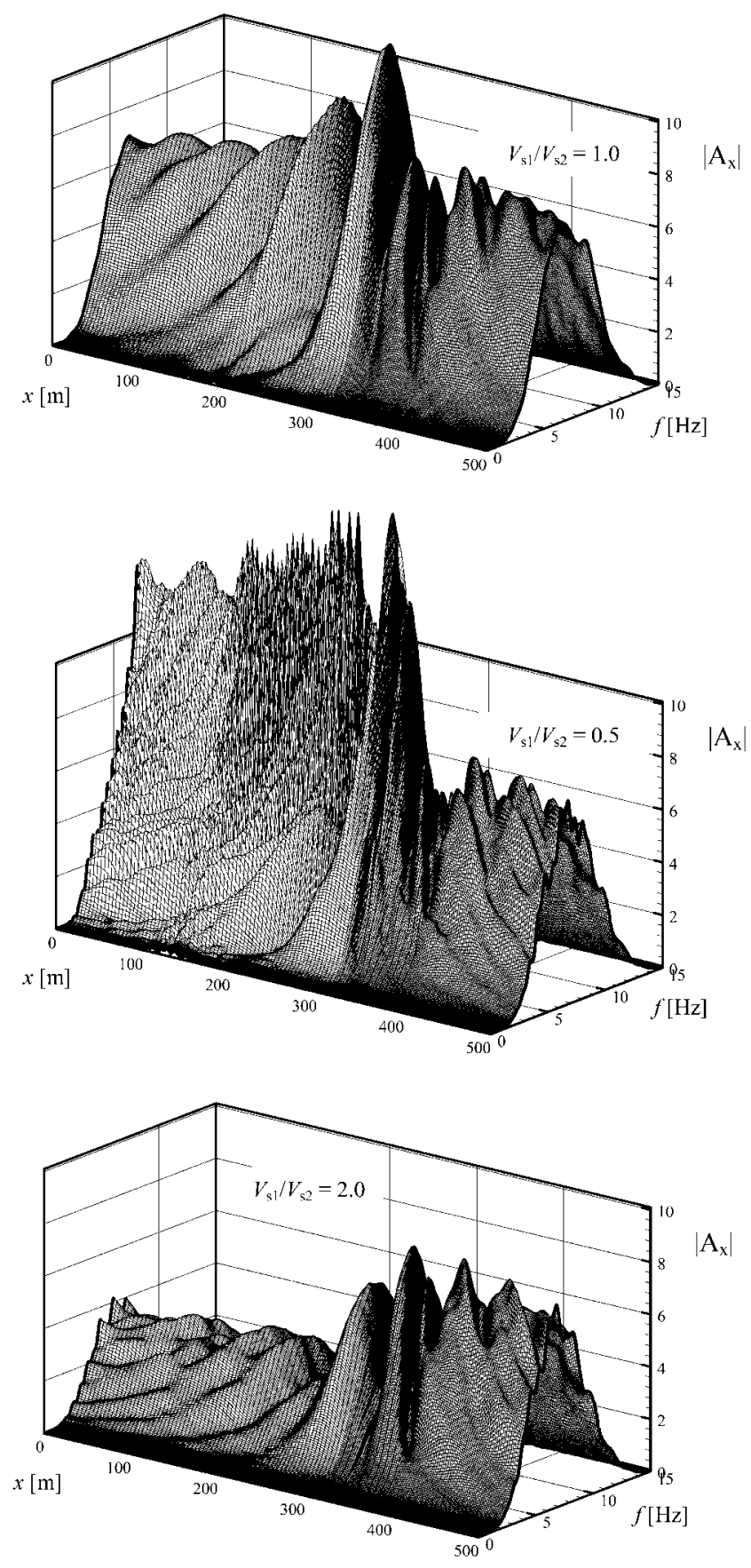

Figure 16. Effect of surface-layer stiffness $\left(V_{s 1} /\right.$ $V_{s 2}$ ) on the Fourier amplitude contours of surface horizontal acceleration for a configuration with $h_{1} /(H-$ $h)=0.25, V_{R} / V_{s 2}=1.0$, and $h / H=0.5$.

and vertical acceleration for typical simulations of the random fields in Figure 17.

The effects of soil randomness on the time- and frequency-domain characteristics of the surface response of clifftype topographies, compared with the corresponding response at the surface of a homogeneous half-space, can be summarized as follows:

1. The duration of the recorded motion at the surface is pro-
Table 3

Parametric Simulations on the Effects of Horizontal Correlation Distance, for $\theta_{z}=2.5 \mathrm{~m}$ and $V_{s, \text { mean }}=200 \mathrm{~m} / \mathrm{sec}$

\begin{tabular}{lrccccc}
\hline & & \multicolumn{5}{c}{$\theta_{\mathrm{x}}(\mathrm{m})$} \\
\cline { 3 - 7 } \multicolumn{1}{c}{$\theta_{x} / \lambda_{0}$} & & 5.0 & 10.0 & 25.0 & 50.0 & 100.0 \\
\hline \multirow{2}{*}{$f_{0}(\mathrm{~Hz})$} & 3.0 & 0.08 & 0.15 & 0.38 & 0.75 & 1.50 \\
& 5.0 & 0.13 & 0.25 & 0.63 & 1.25 & 2.50 \\
& 10.0 & 0.25 & 0.50 & 1.25 & 2.50 & 5.00 \\
\hline
\end{tabular}

Values are shown normalized with the dominant propagating wavelengths of the simulated incident motions.

longed because of multiple reflections/refractions of the incident waves at the localized inhomogeneities of the random medium.

2. The mean value of peak-normalized horizontal acceleration is shown to be slightly reduced because of diffusion of the incident energy toward the crest and subsequent reduction of focusing.

3. The mean amplitude of parasitic acceleration is shown to be slightly enhanced, especially when the horizontal correlation distance is of the same order of magnitude as the dominant propagating wavelengths. Nonetheless, substantial enhancement has been observed for typical realizations, which can be as high as $100 \%$ compared with a homogeneous medium.

4. In the frequency domain, both crest-to-far-field and crestto-base transfer functions of horizontal acceleration are shown to be more erratic than the corresponding transfer functions of a homogeneous medium. In particular, components that correspond to wavelengths of the same order of magnitude as the horizontal correlation distance are shown to be more pronounced.

5. Finally, the frequency-domain characteristics of the vertical component are shown to be significantly affected by the randomness of soil properties. The distribution is substantially more fluctuating, and both low- and highfrequency components are enhanced when compared with the homogeneous medium case.

The amplitude and frequency content of the parasitic response, especially in the high-frequency region of the spectrum, along with the increased duration of response caused by multiple reflections/refractions of the propagating waves, are characteristics of the surface motion that cannot be captured without accounting for the spatial variability of soil properties. These effects may partially explain the observed discrepancy between predictions of theoretical models for homogenous media and weak-motion data, which is particularly evident in the high-frequency components of surface response.

\section{Site Specific Simulations}

We evaluate next the response of multilayered soil profiles with lateral heterogeneity, characteristic of the local 

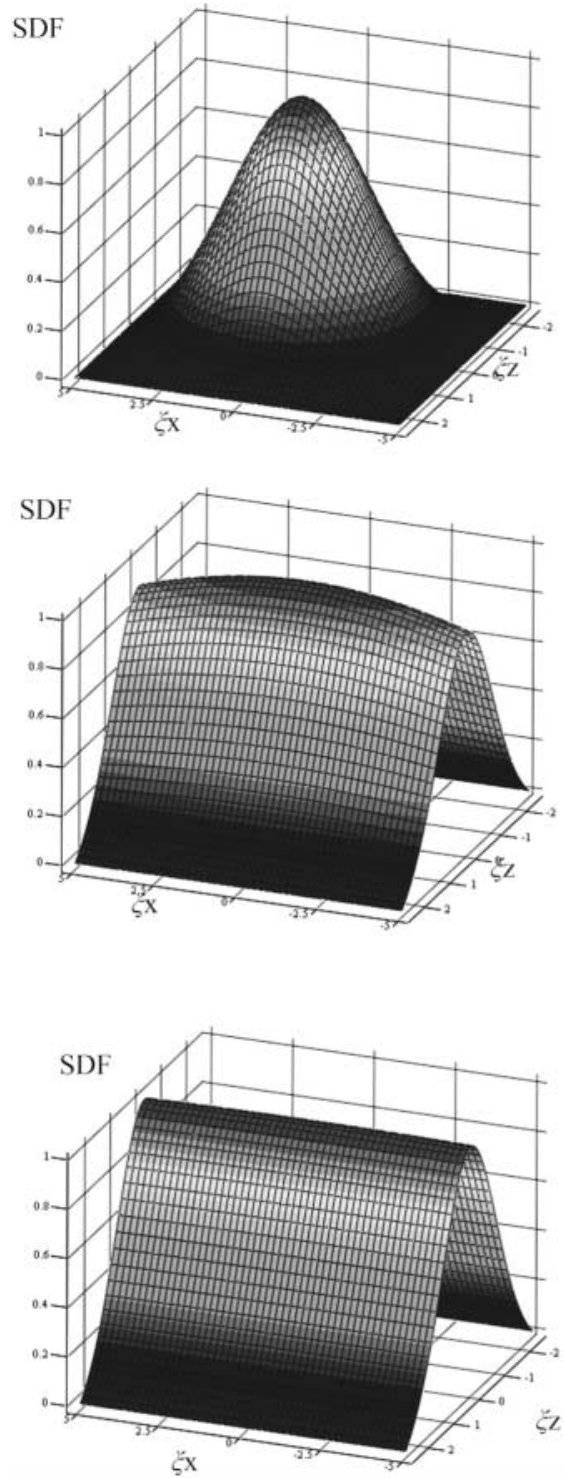

Figure 17. Three-dimensional representation of spectral density functions and typical realizations of the random field with $V_{s \text {,mean }}=200 \mathrm{~m} / \mathrm{sec}$ and $\sigma=30 \mathrm{~m} / \mathrm{sec}$, for various correlation distances.

soil conditions in Adàmes, subjected to typical broadband acceleration recordings from the Athens 1999 event, and we validate our results by comparison with aftershock recordings.

\section{Local Site Conditions}

To construct typical soil profiles in Adàmes, which is necessary for the realization of wave propagation simulations, we compiled geotechnical information from the following sources: (1) geotechnical investigations of the area performed by local agencies and private companies, which comprised 10 boreholes with standard-penetration blowcount $\left(N_{\mathrm{SPT}}\right)$ measurements and limited laboratory information on the variation of plasticity index $\left(I_{p}\right)$ with depth (Eight
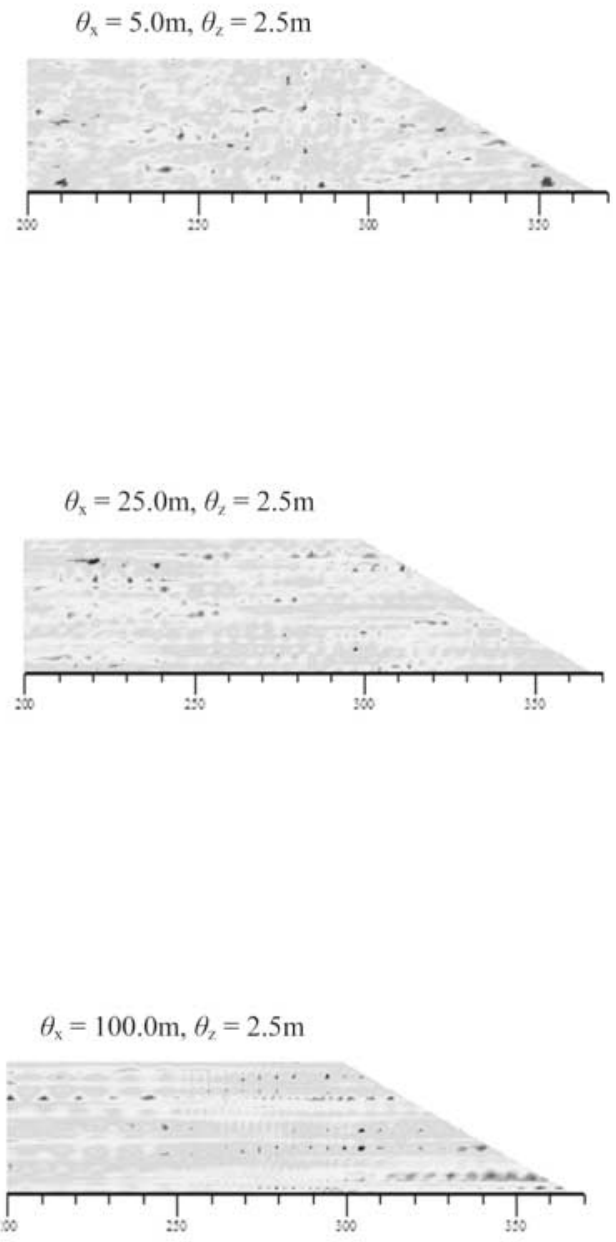

of these had been performed down to a depth of about $35 \mathrm{~m}$, and two had reached almost $80 \mathrm{~m}$.); (2) two 150-m-deep boreholes drilled for the Olympic Village under construction (1.5 km west-northwest of Adàmes) that were used to extrapolate shear-wave velocity profiles at depths greater than $80 \mathrm{~m}$. (3) Cross-hole measurements at four locations along the Kifissos riverbed in the Adàmes area and at scattered distances behind the crest (shear-wave velocity profiles obtained by means of this technique became available at later stages of this study). The location of SPT boreholes and cross-hole measurements is depicted in Figure 3. For further information on the sources of the geotechnical information, see Gazetas et al. (2001).

Because of the scarcity of direct dynamic soil-property measurements, we needed to account for all available geo- 

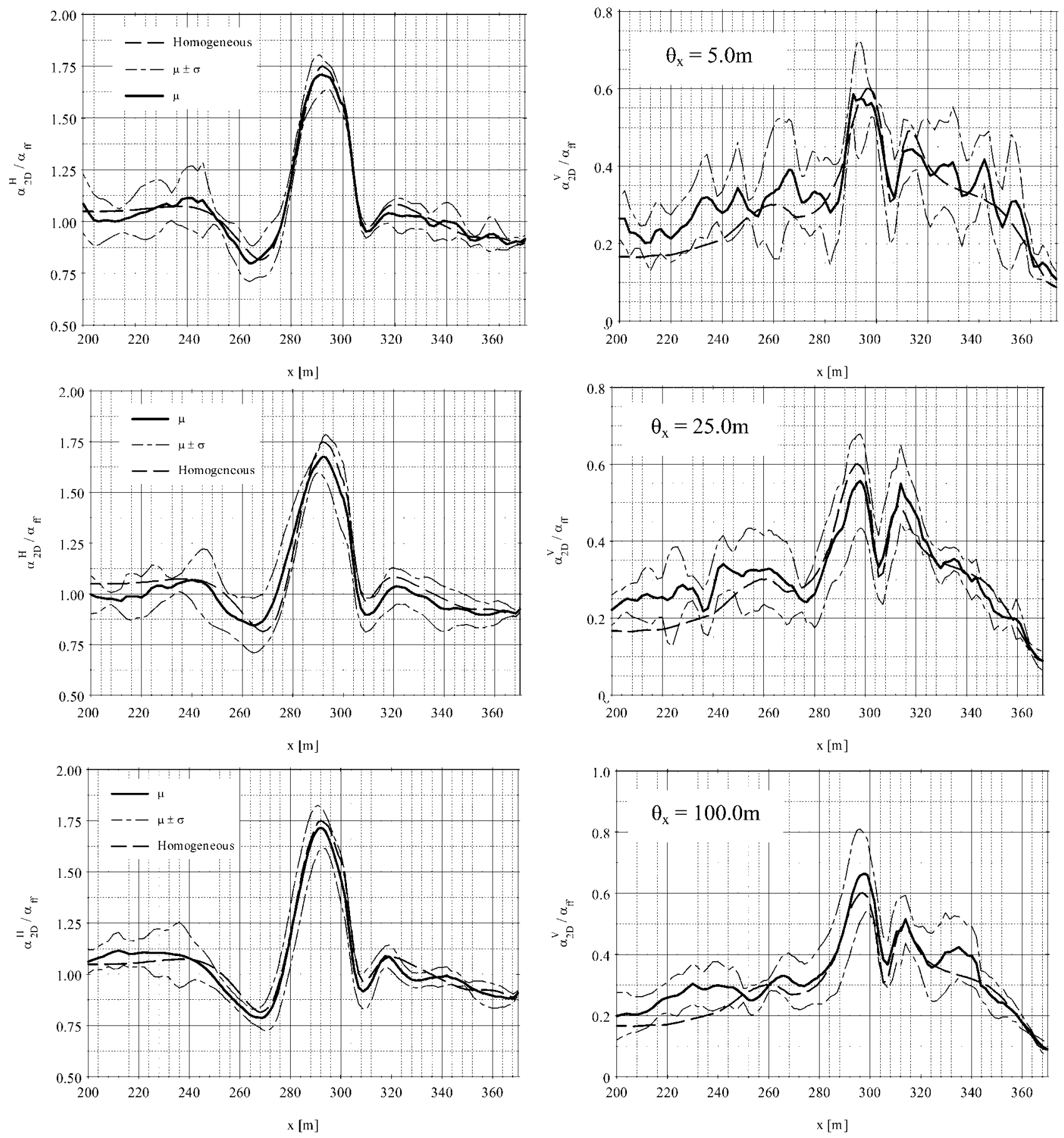

Figure 18. Spatial distribution of peak surface response for the ensemble of numerical simulations. Mean and standard deviation of peak normalized horizontal (left) and vertical (right) acceleration.

technical information simultaneously to construct reliable soil profiles at the site of interest. For this purpose, low-strain shear-wave velocity $\left(V_{s}\right)$ and shear modulus, $G_{\max }$, profiles were also inferred indirectly from the available $N_{\mathrm{SPT}}$ measurements. The empirical correlation selected for this purpose is the one proposed by Imai and Tonuchi (1982):

$$
V_{s}[\mathrm{~m} / \mathrm{sec}]=97.0 N_{\mathrm{SPT}}^{0.314}
$$

Results obtained by means of equation (3) for the shearwave velocity variation with depth were found to be consistent with available cross-hole measurements at the corresponding locations.

Dynamic soil property profiles were finally compiled at three characteristic locations in Adàmes comprising alternating soil layers of silty-gravely sands and sandy-gravely clays in the top 20-30 m; these are referred to in the follow- 

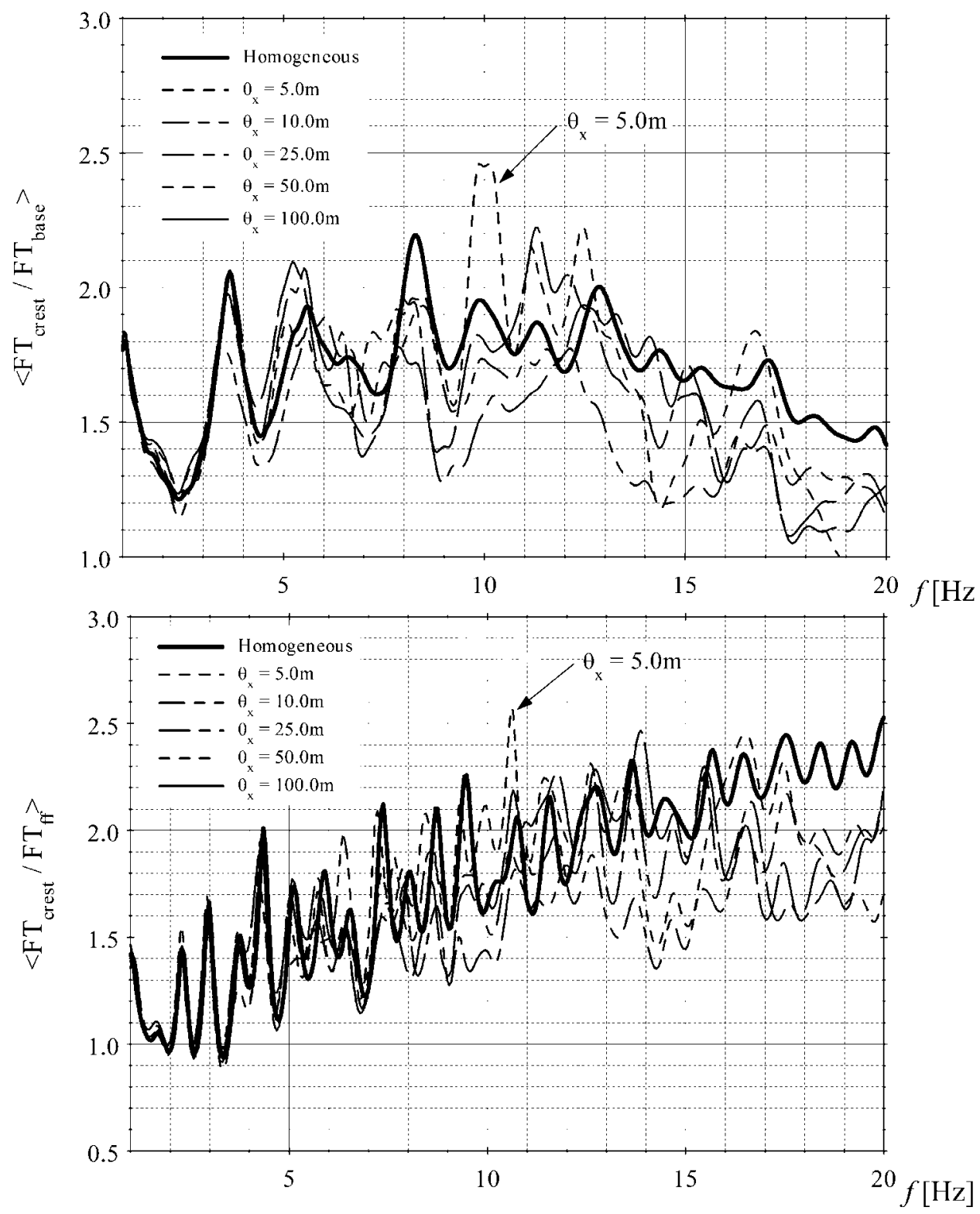

Figure 19. Mean variation of the crest/far-field (left) and crest/base (right) transfer function, for all the correlation distances $\theta_{x}$ investigated.

ing text as profiles A, B, and C. Results of the geotechnical investigation are summarized in Figure 21, where we illustrate the three idealized, horizontally stratified profiles along with the cross-hole measurements at the corresponding locations.

The mean shear-wave velocity and fundamental resonant frequency of the simulated soil profiles are summarized in Table 4 . The quantity $V_{s, 30}$ corresponds to the mean shearwave velocity of the top $30 \mathrm{~m}$ of the profile, used for site characterization in the European Seismic Code (EC8). According to EC8, profiles $\mathrm{A}, \mathrm{B}$, and $\mathrm{C}$ are categorized as very stiff, stiff, and medium stiff, respectively. For each profile, Figure 22 plots the one-dimensional rock outcrop-bedrock transfer functions. The three profiles show substantially different patterns of one-dimensional soil amplification potential.

We performed a local geostatistical analysis to estimate the spatial variability of small-strain stiffness, using both the values extrapolated from the SPT measurements and the cross-hole results. Because of lack of data, the empirical correlation structure in the horizontal direction was evaluated by using the actual distance between boreholes, irrespective of direction. We then simulated the spatial variation of shear-wave velocity by using the stratified profiles A, B, and $\mathrm{C}$ as background media and by superimposing Gaussian stochastic fields with the estimated correlation distances, namely, $\theta_{z}=2.5 \mathrm{~m}$ and $\theta_{x}=15.0 \mathrm{~m}$. For the denormalization of the field, we used a constant standard deviation 
Horizontal Acceleration $\left(\left|\mathbf{A}_{\mathbf{x}}\right|\right)$
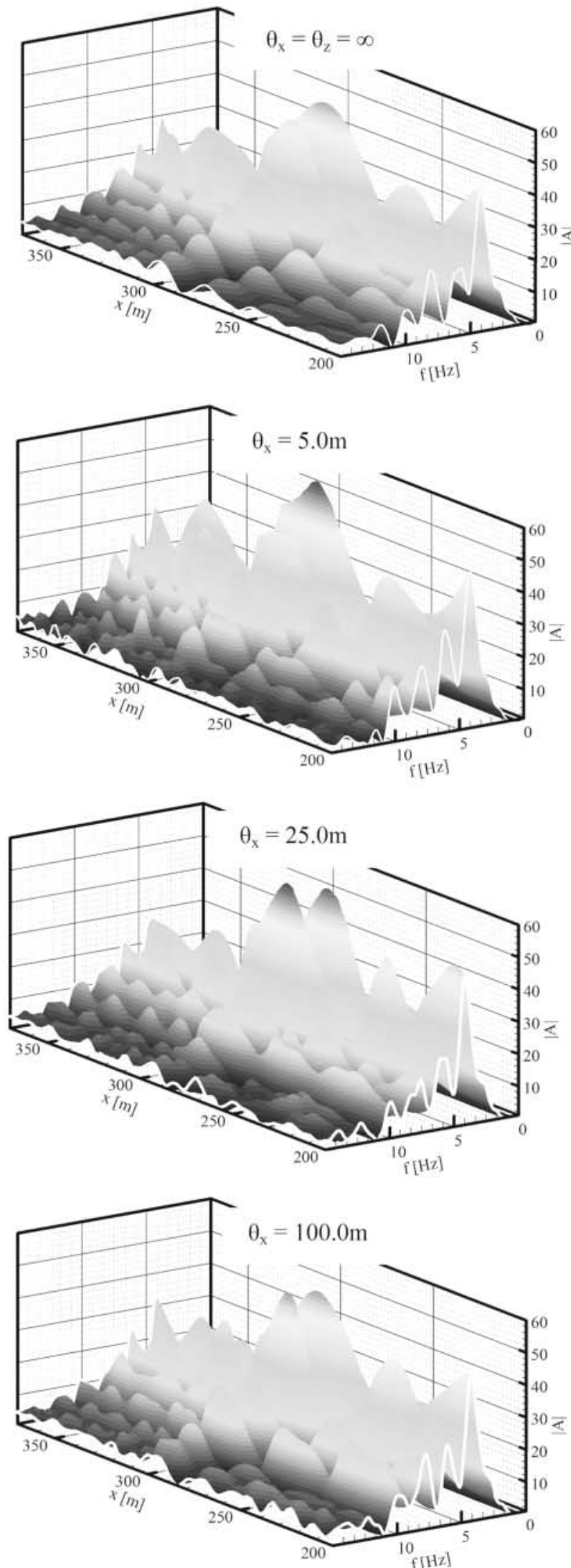

Vertical Acceleration $\left(\left|\mathbf{A}_{z}\right|\right)$
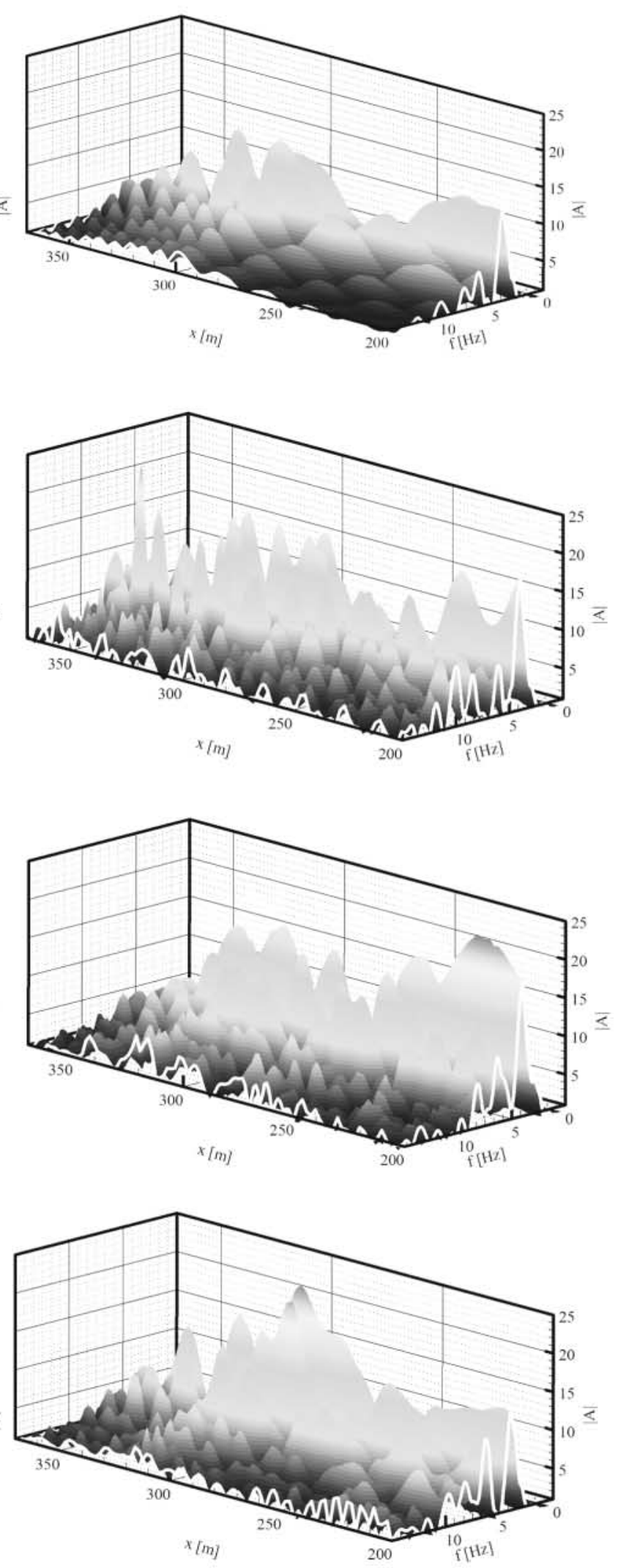

Figure 20. Contours of Fourier spectral amplitude for surface horizontal (left) and vertical (right) response: typical realizations of stochastic field for various correlation distances and incident Ricker wavelets in series, with central frequencies $f_{0}=3,5,10 \mathrm{~Hz}$. 

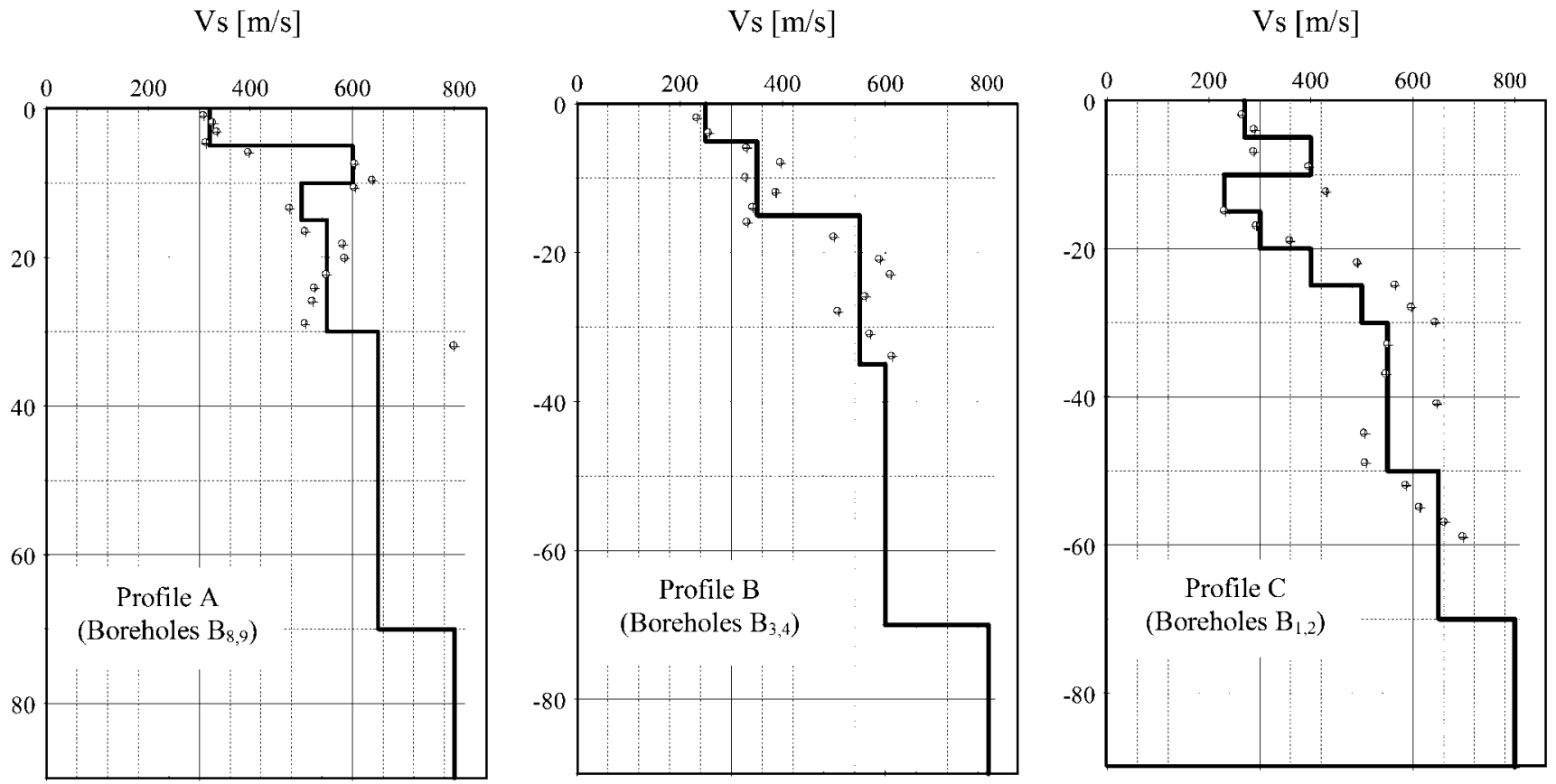

Figure 21. Shear-wave velocity variation with depth for the three characteristic soil profiles $\mathrm{A}, \mathrm{B}$, and $\mathrm{C}$ in Adàmes. The distinct values in the shear-wave velocity graphs correspond to cross-hole shear-wave velocity measured at the corresponding locations. The location of boreholes refers to the notation on Figure 5.

Table 4

Stiffness and Frequency Characteristics of Soil Profiles A, B, and $\mathrm{C}$ in Adàmes

\begin{tabular}{lccc}
\hline & $f_{1}(\mathrm{~Hz})$ & $\bar{V}_{s}(\mathrm{~m} / \mathrm{sec})$ & $\bar{V}_{s, 30}(\mathrm{~m} / \mathrm{sec})$ \\
\hline Profile A & 1.88 & 591 & 489 \\
Profile B & 1.53 & 514 & 395 \\
Profile C & 1.35 & 466 & 326 \\
\hline
\end{tabular}

$\sigma=0.15 V_{s, \text { mean }}$, where $V_{s, \text { mean }}$ is the mean shear-wave velocity of the corresponding profile. The spectral density function and a typical realization of the stochastic field corresponding to profile $\mathrm{C}$ are illustrated in Figure 23.

\section{Broadband Seismic Excitation}

Fifteen strong-motion stations were triggered by the mainshock within $25 \mathrm{~km}$ from the causative fault, providing records of peak ground accelerations (PGA) that range from about $0.05 \mathrm{~g}$ up to $0.50 \mathrm{~g}$. The location of the strongest recorded motions (in terms of PGA) is indicated with filled triangles in Figure 2. Table 5 summarizes the time- and frequency-domain characteristics of these strong-motion time histories (namely, MNSA, SPLB, SGMA, and KEDE), which were used in absence of records in the meizoseismal area. To recover the motion at rock outcropping, numerical one- or two-dimensional deconvolution analyses were performed to remove potential local soil effects and soil- structure interaction. For further details, see Gazetas et al. (2002) and Assimaki and Gazetas (2004).

For the purpose of our study, we first performed wave propagation simulations using Ricker wavelets with central frequency $f_{0}=3 \mathrm{~Hz}$; the frequency content of this pulse, namely $1.5-9.0 \mathrm{~Hz}$, is shown to be similar to that of typical acceleration time histories recorded during the Athens, 1999 event. Subsequently, the aforementioned rock-outcrop acceleration time histories computed from available strongmotion records from the Athens event were used to supplement our numerical simulations by illustrating the frequency-dependent nature of topographic effects for broadband seismic input.

Nonetheless, these four motions were recorded within a narrow region located $10 \mathrm{~km}$ away from the end of the ruptured zone, in a direction perpendicular to it, whereas the area of interest (area e in Fig. 2) lies in front of the rupture zone. Therefore, despite the similar distances of the town of Adàmes and the aforementioned stations from the surface projection of the fault, forward rupture directivity effects that must have been present in the seismic motions experienced by the town of Adàmes during the mainshock could not have been depicted. Subsequent research in the World Data Bank led to the identification of two historic accelerograms from the $1966 M_{\mathrm{S}}$ 5.6 Parkfield, California, earthquake, which encompass simple long-period pulses of strong motion with relatively short duration, indicative of near-fault effects. They were recorded on soft rock at Cholame Shandon no. 8 and Temblor Stations (Leeds 1992). These two records, both 


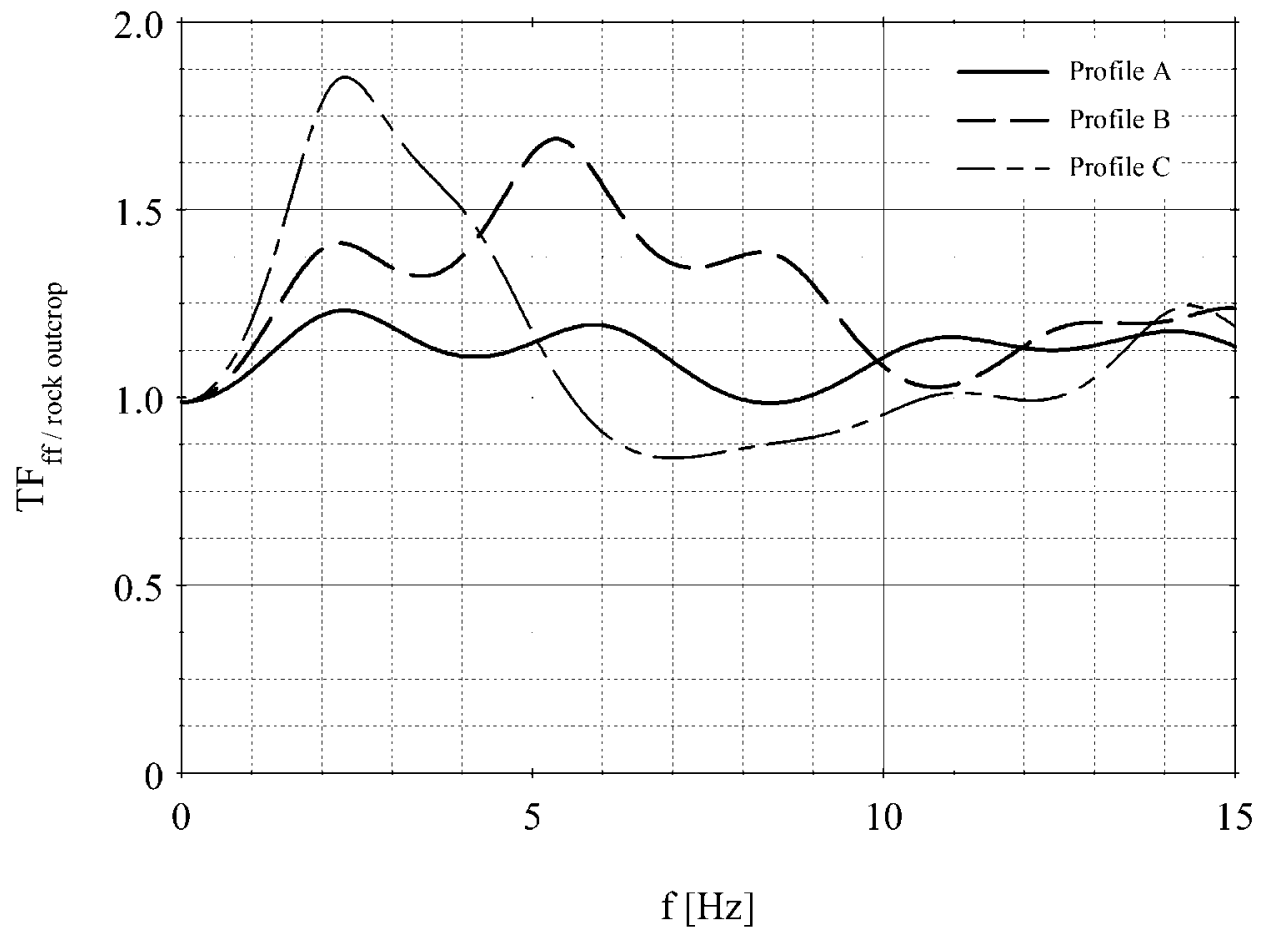

Figure 22. Surface-Bedrock (top) and surface-rock-outcropping (bottom) transfer functions (TF) of the three profiles under investigation: one-dimensional far-field (FF) soil configuration.
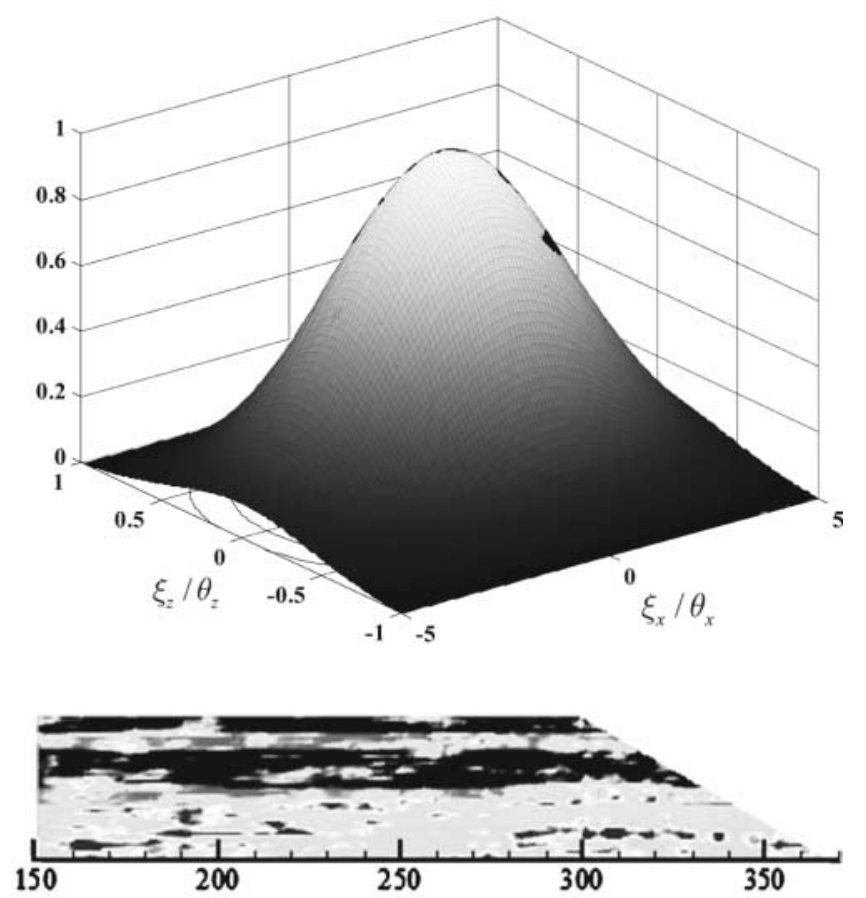

Figure 23. Three-dimensional representation of spectral density function for correlation distances $\theta_{x}=15.0 \mathrm{~m}$ and $\theta_{z}=2.5 \mathrm{~m}$ (top) and typical realization of the shear-wave velocity Gaussian stochastic field corresponding to profile $\mathrm{C}$.
Table 5

Accelerograph Stations That Recorded the Strongest Motions

\begin{tabular}{cccc}
\hline Station Name & Component & $T_{\text {HVSR }}(\mathrm{sec})$ & PGA $(\mathrm{g})$ \\
\hline \multirow{2}{*}{ MNSA } & Long & 0.31 & 0.229 \\
& Trans & 0.20 & 0.512 \\
SPLB & Long & 0.30 & 0.324 \\
& Trans & 0.30 & 0.312 \\
KEDE & Long & 0.24 & 0.264 \\
& Trans & 0.22 & 0.303 \\
SGMA & Long & 0.12 & 0.149 \\
& Trans & 0.16 & 0.239
\end{tabular}

Station locations depicted in Figure 3.

having a PGA of about $0.27 \mathrm{~g}$, resemble the four rock-outcrop motions from the 9 July 1999 event, in the time as well as in the frequency domain.

Accounting for the general strong-motion characteristics of the seismic event by means of the selected mainshock records and for directivity effects, we believe that the ensemble of six time histories used offers a realistic (and perhaps almost complete) description of the free-field base motion for Adàmes. The rock-outcrop acceleration time histories and corresponding response spectra for 5\% structural damping are shown in Figure 24.

Figure 25 illustrates the mean spatial distribution of normalized peak horizontal and vertical acceleration as computed from the ensemble of our numerical simulations, for soil profiles A, B, and C. Our results can be summarized as follows. 

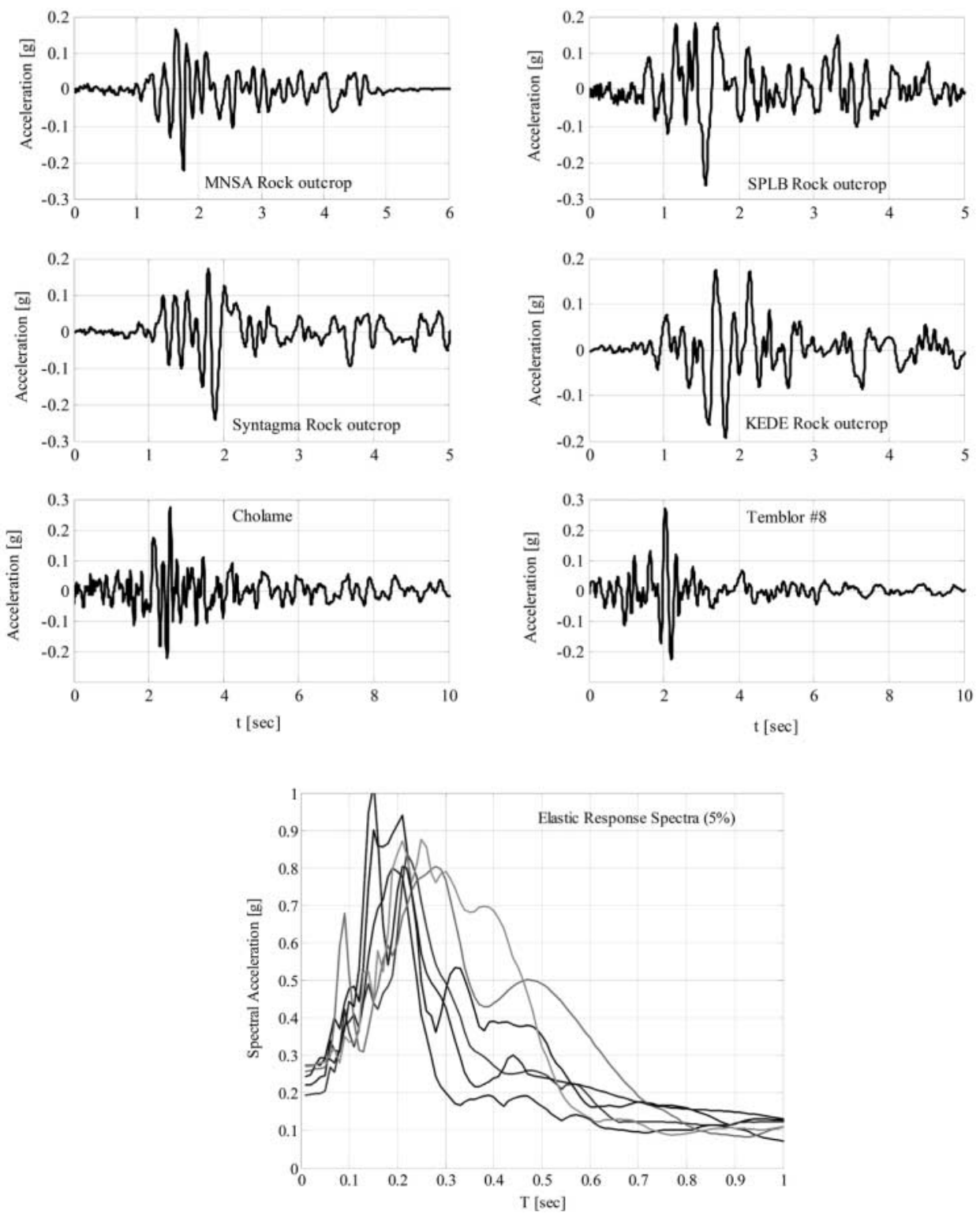

Figure 24. Rock-outcrop acceleration time histories and corresponding elastic response spectra used in our broadband simulations (the MNSA, SGMA, SPLB, and KEDE strong motions from the Athens 1999 mainshock were one- or two-dimensional deconvolved to eliminate site and soil-structure interaction effects, whereas the Cholame and Temblor seismograms were added to the ensemble of motions to simulate forward rupture directivity effects not present in the mainshock recordings).

For the broadband seismic input, topographic amplification occurs within a zone behind the crest, approximately equal to the width of the topographic irregularity $(L=$ $70 \mathrm{~m})$. Frequency components whose wavelengths are of the same order of magnitude as the lateral dimension of the cliff are excessively aggravated because of diffraction and dominate the spatial distribution of surface response. This is in accordance with the results of our parametric investigation.

Topographic aggravation of the horizontal response is shown to be insensitive to soil stratigraphy, yet enhanced in comparison with the homogeneous half-space case. The maximum acceleration is approximately $30 \%$ higher than the corresponding far-field surface response. This again is consistent with the aggravation level computed for $a_{0} \approx 4 h /$ $V_{s, \text { mean }}$ in our parametric study, using as $V_{s \text {,mean }}$ the mean shear-wave velocity of the local soil profile from the surface to the toe of the cliff.

The magnitude of parasitic vertical acceleration, however, shows strong dependence on the soil stratigraphy. This effect is primarily controlled by stiffness of the surface layer. In particular, results show that the amplitude of the vertical acceleration range from $0.25 a_{\mathrm{ff}}$ for the stiffer profile A to 

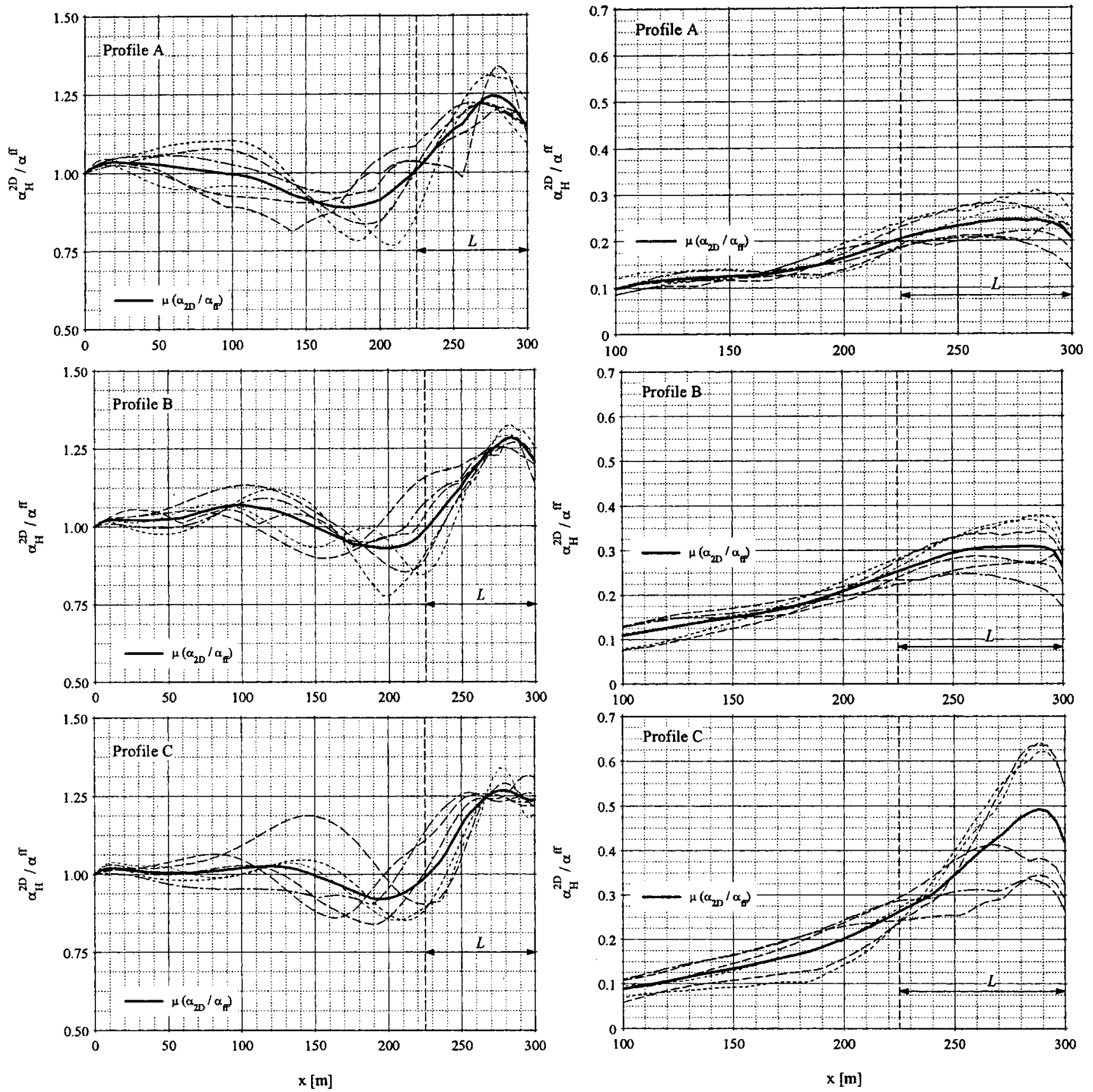

Figure 25. Cumulative results of spatial distribution of the normalized peak horizontal (left) and vertical (right) acceleration along the free surface behind the cliff, for profiles $\mathrm{A}, \mathrm{B}$, and $\mathrm{C}$. (The bold line corresponds to the mean value of the response to the ensemble of input motions.)

$0.70 a_{\mathrm{ff}}$ for the softer profile $\mathrm{C}$, where $a_{\mathrm{ff}}$ is the corresponding far-field peak surface horizontal acceleration.

\section{Recorded Field Evidence}

Significant corroboration of our elastic numerical simulations comes from two sets of ground motions, recorded during two aftershocks of the Athens 1999 event. The instruments were installed in the free field, two at a site $x \approx$
$200 \mathrm{~m}$ from the crest at sites 1 and 2 , and one at $x \approx 20 \mathrm{~m}$ from the crest at site 3; the east-west and north-south unfiltered components recorded on 18 October 1999 at 14:42:54.00 UTC are shown in Figure 26a. The two major aftershocks provided the empirical transfer function spectra illustrated in Figure 26. Because the seismographs were placed at locations with different soil-property characteristics (profile B for the first two and profile $\mathrm{C}$ for the third), the Fourier spectra evaluated from the aftershock accelero- 

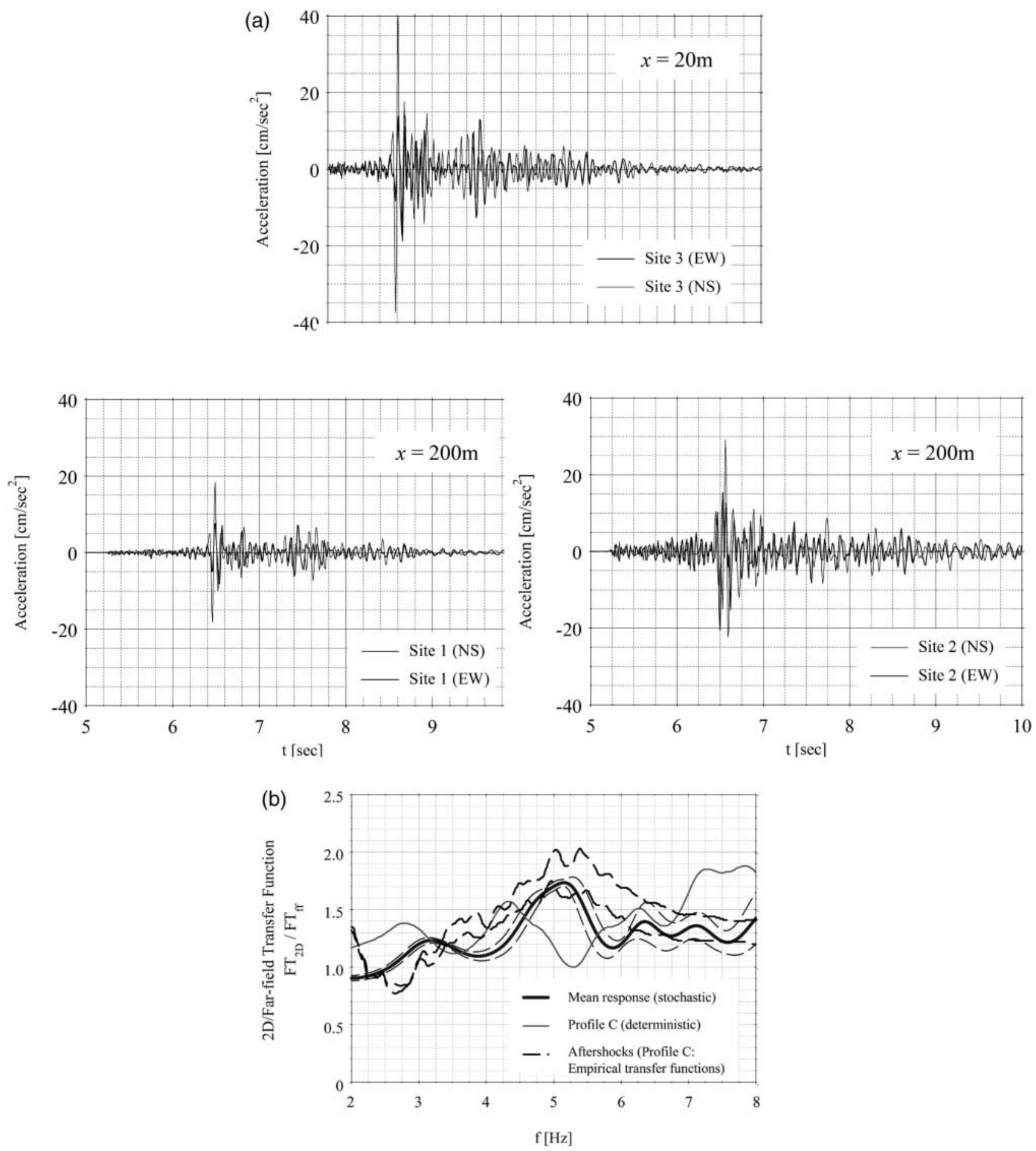

Figure 26. (a) East-West and (north-south unfiltered components recorded on 18 October 1999 at 14:42:54.00 UTC $\left(T_{\text {instr }}=0.019 \mathrm{sec}, \xi_{\text {instr }}=0.66\right)$ at $x=200 \mathrm{~m}$ (sites 1 and 2) and $x=20 \mathrm{~m}$ (site 3 ) from the crest. (b) Two-dimensional/far-field empirical transfer function from the records of two strong aftershocks and comparison with the numerical results of 20 Gaussian stochastic field realizations. 
grams have been initially divided by the one-dimensional transfer function for each profile. Because the recorded peak accelerations were on the order of $0.015 \mathrm{~g}$, we used the lowstrain dynamic soil properties for this purpose. Thus, the variability arising from soil-column flexibility effects has been eliminated. The two major aftershocks provided the empirical transfer function spectra shown in Figure 26.

Also plotted in the Figure 26 is the transfer function computed for the horizontally stratified soil profile corresponding to profile $\mathrm{C}$ by using a Ricker wavelet with central frequency $f_{0}=5 \mathrm{~Hz}$. The frequency content of this incident motion is similar to that of the aftershock recordings, yet the distribution of topographic aggravation in the frequency domain is not considered satisfactory.

The successful class A prediction shown in Figure 26 was achieved by using the complete description of local soil conditions, namely the stratigraphy and heterogeneity of profile C. Note that, because the aftershock recordings were obtained at site 3 (see Fig. 3), where the underlying soil profile corresponds to profile $\mathrm{C}$, the stochastic field is generated based on the stiffness variation of this profile with depth.

We here illustrate the mean and standard deviation of the numerically predicted transfer functions from 40 realizations of the stochastic field at $x=20 \mathrm{~m}$. For these simulations, a Ricker wavelet with central frequency $f_{0}=5 \mathrm{~Hz}$ was also used. The recorded and computed results are clearly in very good agreement, offering strong support to our observations.

\section{Conclusions}

An extensive parametric study has been conducted to evaluate the effects of local soil conditions on the diffraction mechanism near the vertex of clifftype topographies during the 1999 Athens earthquake. In particular, we focused on the vicinity of the Kifissos river canyon, a location at which excessive structural damage was reported. Simulations were carried out for a homogeneous soil layer overlaying elastic bedrock, a two-layered soil profile, and a random medium generated by means of Gaussian probability distribution functions.

We have shown that soil horizontal layering and lateral heterogeneity of the soil profile play a significant role in the topographic aggravation of seismic motion next to the crest, namely the amplification of surface response with respect to the far field. In particular, (1) transmitted and reflected waves at the boundaries of soil layers, (2) trapped waves within softer surface formations, and (3) scattered waves at localized patches of material, with strong impedance contrast compared with the background medium, interact with the diffracted wave field that is directed by the geometry of the topographic irregularity. The effects of local soil conditions have been shown to affect the spatial distribution, aggravation level, and frequency content of the surface response next to the crest.
Using a case study from the Athens 1999 earthquake, we have shown that, despite the detrimental diffraction potential of the cliff, geometry alone could not explain the degree of topographic aggravation of seismic motion. We have shown that the role of topography strongly depends on the underlying soil conditions, thus justifying the highly nonuniform damage distribution in a town of rather uniform structural quality, located along the vertex of a practically two-dimensional topographic feature. In particular, for profile $\mathrm{C}$, the presence of a distinct soft surface layer excessively enhanced the amplitude of parasitic vertical acceleration in comparison with profiles $\mathrm{A}$ and $\mathrm{B}$, partially explaining the severity of structural damage at this location.

Finally, we have illustrated that weak-motion data can be used successfully as a valuable guide in reconnaissance studies. Using a complete description of local soil conditions, namely soil stratigraphy and material heterogeneity, our results are in very good agreement with recorded aftershocks from the event, strengthening the validity of our conclusions.

Nonetheless, our studies have also shown that weakmotion data may not be applicable to describing the topographic effects associated with strong ground motion, usually associated with nonlinear soil behavior. Simulations and conclusions concerning topographic effects for seismic excitations strong enough to cause observable inelastic effects shall be taken up in a forthcoming paper.

\section{References}

Aki, K. (1980). Attenuation of shear waves in the lithosphere for frequencies from 0.05 to $25 \mathrm{~Hz}$, Phys. Earth Planet. Interiors 21, 50-60.

Aki, K. (1988). Local site effects on strong ground motion, in Earthquake Engineering and Soil Dynamics II, vol. 20, Geotechnical Special Publication, J. V. Thun (Editor), American Society of Civil Engineers, New York, 103-155.

Ashford, S. A., and N. Sitar (1997). Analysis of topographic amplification of inclined shear waves in a steep coastal bluff, Bull. Seism. Soc. Am. 87, 692-700.

Ashford, S. A., N. Sitar, J. Lysmer, and N. Deng (1997). Topographic effects on the seismic response of steep slopes, Bull. Seism. Soc. Am. 87, no. 3, 701-709.

Assimaki, D., and G. Gazetas (2004). Soil and topographic amplification on canyon banks and the Athens 1999 earthquake, J. Earthquake Eng. 8, no. 1, 1-44.

Bard, P. Y. (1982). Diffracted waves and displacement field over two dimensional elevated topographies, Geophys. J. R. Astr. Soc. 71, 731760.

Bard, P. Y. (1999). Local effects on strong ground motion: physical basis and estimation methods in view of microzoning studies, in Proc. Advanced Study Course "Seismotectonic and Microzonation Techniques in Earthquake Engineering," Kefallinia, Greece, 1999, 4 September $127-218$.

Bard, P. Y., and B. E. Tucker (1985). Ridge and tunnel effects: comparing observations with theory, Bull. Seism. Soc. Am. 75, 905-922.

Boore, D. M (1972). Note on the effect of topography on seismic SH waves, Bull. Seism. Soc. Am. 62, 275-284.

Boore, D. M., S. C. Harmsen, and S. T. Harding (1981). Wave scattering from a step change in surface topography, Bull. Seism. Soc. Am. 71, no. $1,117-125$. 
Bouchon, M. (1973). Effect of topography on surface motion, Bull. Seism. Soc. Am. 63, 615-632.

Bouchon, M., and J. S. Barker (1996). Seismic response of a hill: the example of Tanzana, California, Bull. Seism. Soc. Am. 86, 66-72.

Bouchon, M., C. A. Schultz, and M. N. Toksoz (1995). Effect of 3D topography on seismic motion, J. Geophys. Res. 10, no. B3, 5835-5846.

Buchbinder, G. G. R., and R. A. W. Haddon (1990). Azimuthal anomalies of short-period P-wave arrivals from Nahanni aftershocks, northwest territories, Canada, and effects of surface topography, Bull. Seism. Soc. Am. 80, no. 5, 1272-1283.

Celebi, M. (1987). Topographical and geological amplifications determined from strong-motion and aftershock records of the 3 March 1985 Chile earthquake, Bull. Seism. Soc. Am. 77, 1147-1157.

Davis, L. L., and R. West (1973). Observed effects of topography on ground motion, Bull. Seism. Soc. Am. 63, 283-298.

Delibasis, N., P. Papadimitriou, N. Voulgaris, and I. Kassaras (2000). The Parnitha fault: a possible relationship with other neighboring faults and causes of larger damages, Ann. Geol. Pays Hell. 1e serie, T. XXXVIII, Fasc, B, 41-50.

Deng, X. (1991). The integrated adjustment of 3-D terrestrial network and GPS observations, Master's Thesis, Wuhan Technical University of Surveying and Mapping.

European Seismic Code (EC8) (2000). Design provisions for earthquake resistance of structures, Part 1-1: General rules-seismic actions and general requirements for structures (Draft), prEN 1998-5, European Committee for Standardization, Brussels.

England, R., F. J. Sabina, and I. Herrera (1980). Scattering of $S H$ waves by surface cavities of arbitrary shape using boundary methods, Phys. Earth Planet. Interiors 21, 148-157.

Flatté, S. M., and R. S. Wu (1988). Small-scale structure in the lithosphere and asthenosphere deduced from arrival-time and amplitude fluctuations at NORSAR, J. Geophys. Res. 93, 6601-6614.

Gazetas, G. (1996). Soil Dynamics and Earthquake Engineering: Case Histories, Simeon Press, Athens (in Greek).

Gazetas Associates, Consulting Engineers (2001). Computational and experimental assessment of strong ground motion within the meizoseismal area of the Parnitha, 7-9-99, earthquake, Technical Report, Earthquake Planning and Protection Organization, OASP, p. 207 (in Greek).

Gazetas, G., P. Dakoulas, and A. S. Papageorgiou (1990). Local soil and source mechanism effects in the 1986 Kalamata, Greece, earthquake, Earthquake Eng. Struct. Dyn. 19, 431-456.

Gazetas, G., P. V. Kallou, and P. N. Psarropoulos (2002). Topography and soil effects in the $\mathrm{M}_{\mathrm{S}}$ 5.9 Parnitha (Athens) earthquake: the case of Adàmes, Nat. Hazards 27, no. 1-2, 133-169.

Geli, L., P.-Y. Bard, and B. Jullien (1988). The effect of topography on earthquake ground motion: a review and new results, Bull. Seism. Soc. Am. 78, 42-63.

Gilbert, F., and L. Knopoff (1960). Seismic scattering from topographic irregularities, J. Geophys. Res. 65, 3437-3444.

Griffiths, D. W., and G. A. Bollinger (1979). The effect of Appalachiam mountain topography on seismic waves, Bull. Seism. Soc. Am. 69, $1081-1105$.

Hudson, J. A. (1967). Scattered surface waves from a surface obstacle, Geophys. J. R. Astr. Soc. 13, 441-458.

Hudson, J. A., and D. M. Boore (1980). Comments on scattered surface waves from a surface obstacle, Geophys. J. R. Astr. Soc. 60, 123-127.

Kawase, H., and K. Aki (1990). Topography effect at the critical SV wave incidence: possible explanation of damage pattern by to the WhittierNarrows, California, Earthquake of 1 October 1987, Bull. Seism. Soc. Am. 80, 1-22.

Kontoes, C., P. Elias, O. Sykioti, P. Briole, D. Remy, M. Sachpazi, G. Veis, and I. Kotsis (2000). Displacement field and fault model for the September 7, 1999 Athens earthquake inferred from ERS2 satellite radar interferometry, Geophys. Res. Lett. 27, no. 24, 3989-3992.

Lebrun, B., D. Hatzfeld, P.-Y. Bard, and M. Bouchon (1999). Experimental study of the ground motion on a large scale topography hill at Kitherion (Greece), J. Seism. 3, 1-15.

Nechtschein, S., P.-Y. Bard, J.-C. Gariel, J.-P. Mèneroud, P. Dervin, M. Cushing, B. Gaubert, S. Vidal, and A. M. Duval (1995). A topographic effect study in the Nice region, in Proceedings of the 5th International Conference on Seismic Zonation, 17-19 October 1995, Nice, France, 1067-1074.

Ohtsuki, A., and K. Harumi (1983). Effect of topography and subsurface inhomogeneities on seismic SV waves, Earthquake Eng. Struct. Dyn. 11, 441-462.

Papadimitriou, P., N. Voulgaris, I. Kassaras, N. Delibasis, and K. Makropoulos (2000). The September 7, 1999 Athens earthquake sequence recorded by the Cornet network: preliminary results of source parameter determination of the mainshock, Ann. Geol. Pays Hell. 1 serie, T. XXXVIII, Fasc, B, 29-40.

Pavlides, S. B., G. Papadopoulos, and A. Ganas (2002). The fault that caused the Athens September $1999 \mathrm{M}_{\mathrm{S}}=5.9$ earthquake: field observations, Nat. Hazards 27, no. 1-2, 1-25.

Pedersen, H., B. LeBrun, D. Hatzfeld, M. Campillo, and P.-Y. Bard (1994). Ground motion amplitude across ridges, Bull. Seism. Soc. Am. 85, $1786-1800$.

Popescu, R. (1995). Stochastic variability of soil properties: data analysis, digital simulation, effects on system behavior, Ph.D. thesis, Princeton University, Princeton, New Jersey.

Popescu, R., J. H. Prevost, and G. Deodatis (1998a). Spatial variability of soil properties: two case studies. Geotech. Earthquake Eng. Soil Dynamics III, American Society of Civil Engineers, 568-579.

Prevost, J. H. (1989). DYNA1D: a computer program for nonlinear seismic site response analysis, Technical rept. NCEER-89-0025, Department of Civil Engineering and Operations Research, Princeton University, Princeton, New Jersey.

Prevost, J. H. (1993). Nonlinear dynamic response analysis of soil and soilstructure interacting systems, in Soil Dynamics and Geotechnical Earthquake Engineering, P. Seco e Pinto (Editor), Balkema, Rotterdam, the Netherlands, 49-126.

Psycharis, I., D. Papastamatiou, I. Taflambas, P. Carydis, G. Bouckovalas, G. Gazetas, I. Kalogeras, G. Stavrakakis, S. Pavlides, E. Lekkas, C. Kranis, C. Ioannidis, C. Cholevas, and D. Pyrros (1999). The Athens, Greece Earthquake of September 7, 1999, Reconnaissance report published on the Earthquake Engineering Research Institute (EERI), Newsletter: Special Earthquake Report, November.

Rogers, A. M., L. J. Katz, and T. J. Bennett (1974). Topographic effect on ground motion for incident P waves: a model study, Bull. Seism. Soc. Am. 64, 437-456.

Rondogianni, Th., A. Mettos, D. Galanakis, and Ch. Georgiou (2000). The Athens earthquake of September 7, 1999: its setting and effects, Ann. Geol. Pays Hell. 1e serie, T. XXXVIII, Fasc, B, 131-144.

Sanchez-Sésma, F. J. (1983). Diffraction of elastic waves by three-dimensional surface irregularities, Bull. Seism. Soc. Am. 73, 1621-1636.

Sanchez-Sésma, F. J. (1990). Elementary solutions for the response of a wedge-shaped medium to incident SH and SV waves, Bull. Seism. Soc. Am. 80, 737-742.

Sanchez-Sésma, F. J., and M. Campillo (1991). Diffraction of P, SV and Rayleigh waves by topographic features. A boundary integral formulation, Bull. Seism. Soc. Am. 81, no. 6, 2234-3353.

Sanchez-Sèsma, F. J., and E. Rosenblueth (1979). Ground motion at canyons of arbitrary shape under incident SH waves, Int. J. Earthquake Eng. Struct. Dyn. 7, 441-450.

Sato, H. (1979). Wave propagation in one-dimensional inhomogeneous elastic media, J. Phys. Earth 27, 455-466.

Sato, H. (1982). Attenuation of S waves in the lithosphere due to scattering by its random velocity structure, J. Geophys. Res. 87, 7779-7785.

Sato, H., and M. Fehler (1998). Seismic Wave Propagation and Scattering in the Heterogeneous Earth, AIP Press/Springer Verlag, New York, $308 \mathrm{pp}$.

Schultze, E. (1971). Frequency distributions and correlations of soil prop- 
erties, in Proc. 1st International Conf. Appl. Stat. Prob. Soil Struct. Eng., Hong Kong, 13-16 September 1971, 371-387.

Shinozuka, M., and G. Deodatis (1996). Simulation of multidimensional Gaussian stochastic fields by spectral representation, App. Mech. Reviews 49, no. 1, 29-53.

Sills, L. (1978). Scattering of horizontally polarized shear waves by surface irregularities, Geophys. J. R. Astr. Soc. 54, 319-348.

Smith, W. D. (1975). The application of finite element analysis to body wave propagation problems, Geophys. J. 42, 747-768.

Soong, T. T., and M. Grigoriu (1993). Random Vibrations of Mechanical and Structural Systems, Pentice Hall, New York.

Stavrakakis, G. (1999). The Athens Earthquake of September 7, 1999, Newsletter of the European Centre on Prevention and Forecasting of Earthquakes 3, 26-29.

Trifunac, M. D. (1973). Scattering of plane SH waves by a semi-cylindrical canyon, Int. J. Earthquake Eng. Struct. Dyn. 1, 267-281.

Tselentis, G. A., and J. Zahrandnik (2000a). Aftershock monitoring of the Athens Earthquake of 7 September 1999, Seism. Res. Lett. 71, no. 3, 330-337.

Tselentis, G. A., and J. Zahrandnik (2000b). The Athens earthquake of 7 September 1999, Bull. Seism. Soc. Am. 90, no. 5, 1143-1160

Tucker, B. E., J. L. King, D. Hatzfeld, and I. L. Nersesov (1984). Observations of hard rock site effects, Bull. Seism. Soc. Am. 74, 121-136.

Umeda, K., M. Kusanagi, A. Furusawa, and T. Ui (1996). Holocene eruptive activity of Yufu-Tsurumi volcano group found from piston cores in Beppu Bay, Central Kyushu, Bull. Volcanol. Soc. Jpn. 41, 61-71 (in Japanese with English abstract).

Vanmarcke, E. H. (1983). Random Fields: Analysis and Synthesis, The MIT Press, Cambridge, Massachusetts.
Wong, H. L., and P. C. Jennings (1975). Effect of canyon topography on strong ground motion, Bull. Seism. Soc. Am. 72, 1167-1183.

Wong, H. L., and M. D. Trifunac (1974). Scattering of plane SH waves by a semi-elliptical canyon, Int. J. Earthquake Eng. Struct. Dyn. 3, 157169.

Wu, R.-S. (1982). Attenuation of short period seismic waves due to scattering, Geophys. Res. Lett. 9, no. 1, 9-12.

Institute for Crustal Studies

Mail Code 1100, 1140 Girvetz Hall

University of California

Santa Barbara, California 93106-1100

dominic@crustal.ucsb.edu

(D.A.)

Department of Civil Engineering-Geotechnical Divison

National Technical University of Athens

2 Giavassi Street

Agia Paraskeui, 15342 Athens, Greece

gazetas@ath.forthnet.gr

(G.G.)

Department of Civil and Environmental Engineering (Rm. 1-271)

Massachusetts Institute of Technology

77 Massachusetts Avenue

Cambridge, Massachusetts 02139

kausel@mit.edu

(E.K.)

Manuscript received 1 April 2004. 